\title{
REGULARIZATION PROPERTIES OF THE 2D HOMOGENEOUS BOLTZMANN EQUATION WITHOUT CUTOFF
}

\author{
VLAD BALLY AND NICOLAS FOURNIER
}

\begin{abstract}
We consider the 2-dimensional spatially homogeneous Boltzmann equation for hard potentials. We assume that the initial condition is a probability measure that has some exponential moments and is not a Dirac mass. We prove some regularization properties: for a class of very hard potentials, the solution instantaneously belongs to $H^{r}$, for some $r \in(-1,2)$ depending on the parameters of the equation. Our proof relies on the use of a well-suited Malliavin calculus for jump processes.
\end{abstract}

\section{INTRODUCTION}

The Boltzmann equation. We consider a spatially homogeneous gas in dimension 2 modeled by the Boltzmann equation. The density $f_{t}(v)$ of particles with velocity $v \in \mathbb{R}^{2}$ at time $t \geq 0$ solves

$$
\partial_{t} f_{t}(v)=\int_{\mathbb{R}^{2}} d v_{*} \int_{-\pi / 2}^{\pi / 2} d \theta B\left(\left|v-v_{*}\right|, \theta\right)\left[f_{t}\left(v^{\prime}\right) f_{t}\left(v_{*}^{\prime}\right)-f_{t}(v) f_{t}\left(v_{*}\right)\right]
$$

where

$$
v^{\prime}=\frac{v+v_{*}}{2}+R_{\theta}\left(\frac{v-v_{*}}{2}\right), \quad v_{*}^{\prime}=\frac{v+v_{*}}{2}-R_{\theta}\left(\frac{v-v_{*}}{2}\right)
$$

and where $R_{\theta}$ is the rotation of angle $\theta$. One usually integrates $\theta$ on $(-\pi, \pi)$, but a famous trick allows one to restrict the integration to $[-\pi / 2, \pi / 2]$ without loss of generality, see e.g. the argument in the introduction of [1]. The cross section $B\left(\left|v-v_{*}\right|, \theta\right) \geq 0$ is given by physics and depends on the type of interaction between particles. We refer to the review paper of Villani [16] for more details. Conservation of mass, momentum and kinetic energy hold for reasonable solutions to (1.1):

$$
\forall t \geq 0, \quad \int_{\mathbb{R}^{2}} f_{t}(v) \psi(v) d v=\int_{\mathbb{R}^{2}} f_{0}(v) \psi(v) d v, \quad \psi=1, v,|v|^{2}
$$

and we classically may assume without loss of generality that $\int_{\mathbb{R}^{2}} f_{0}(v) d v=1$ and $\int_{\mathbb{R}^{2}} v f_{0}(d v)=0$.

Assumptions. We shall assume here that for some $\gamma \in(0,1), \nu \in(0,1 / 2)$, some even function $b:[-\pi / 2, \pi / 2] \backslash\{0\} \mapsto \mathbb{R}_{+}$,

$$
(\mathbf{A}(\gamma, \nu)) \quad\left\{\begin{array}{l}
B\left(\left|v-v_{*}\right|, \theta\right)=\left|v-v_{*}\right|^{\gamma} b(\theta), \quad c \theta^{-1-\nu} \leq b(\theta) \leq C \theta^{-1-\nu}, \\
\exists 0<c<C, \quad \forall \theta \in(0, \pi / 2], \quad c \theta^{-1}, \quad \forall \theta \in(0, \pi / 2], \quad\left|b^{(k)}(\theta)\right| \leq C_{k} \theta^{-1-\nu-k} . \\
\forall k \geq 1, \quad \exists C_{k}, \quad \forall \theta \text {. }
\end{array}\right.
$$

This assumption is made by analogy to the case where particles collide by pairs due to a repulsive force proportional to $1 / r^{s}$ for some $s>2$ in dimension 3 , for which $\gamma=(s-5) /(s-1)$ and

2000 Mathematics Subject Classification. 60H07,82C40.

Key words and phrases. Kinetic equations, Hard potentials without cutoff, Malliavin calculus, Jump processes.

Le second auteur de ce travail a bénéficié d'une aide de l'Agence Nationale de la Recherche portant la référence ANR-08-BLAN-0220-01. 
$b(\theta) \simeq|\theta|^{-1-\nu}$, with $\nu=2 /(s-1)$. We aim to study here hard potentials $(s>5)$, for which $\gamma \in(0,1)$ and $\nu \in(0,1 / 2)$.

Weak solutions. For $\theta \in(-\pi / 2, \pi / 2)$, we introduce

$$
A(\theta)=\frac{1}{2}\left(R_{\theta}-I\right)=\frac{1}{2}\left(\begin{array}{cc}
\cos \theta-1 & -\sin \theta \\
\sin \theta & \cos \theta-1
\end{array}\right) .
$$

Note that $v^{\prime}=v+A(\theta)\left(v-v_{*}\right)$ and that for $X \in \mathbb{R}^{2}$,

$$
|A(\theta) X|^{2}=\frac{1}{2}(1-\cos \theta)|X|^{2} \leq \frac{\theta^{2}}{4}|X|^{2}
$$

Definition 1.1. Assume $(\mathbf{A}(\gamma, \nu))$ for some $\nu \in(0,1)$ and $\gamma \in(0,1]$. A family $\left(f_{t}\right)_{t \in[0, T]}$ of probability measures on $\mathbb{R}^{2}$ is said to be a weak solution of (1.1) if for all $t \in[0, T]$,

$$
\int_{\mathbb{R}^{2}} v f_{t}(d v)=\int_{\mathbb{R}^{2}} v f_{0}(d v) \quad \text { and } \quad \int_{\mathbb{R}^{2}}|v|^{2} f_{t}(d v)=\int_{\mathbb{R}^{2}}|v|^{2} f_{0}(d v)<\infty
$$

and if for any $\psi: \mathbb{R}^{2} \mapsto \mathbb{R}$ globally Lipschitz continuous and any $t \in[0, T]$,

(1.4) $\frac{d}{d t} \int_{\mathbb{R}^{2}} \psi(v) f_{t}(d v)=\int_{\mathbb{R}^{2}} f_{t}(d v) \int_{\mathbb{R}^{2}} f_{t}\left(d v_{*}\right) \int_{-\pi / 2}^{\pi / 2} b(\theta) d \theta\left|v-v_{*}\right|^{\gamma}\left[\psi\left(v+A(\theta)\left(v-v_{*}\right)\right)-\psi(v)\right]$.

The right hand side of (1.4) is well-defined due to (1.3), (1.2) and because $\int_{-\pi / 2}^{\pi / 2}|\theta| b(\theta) d \theta<\infty$ thanks to $(\mathbf{A}(\gamma, \nu))$ with $\nu \in(0,1)$. As shown in [10, Corollary 2.3 and Lemma 4.1], we have the following result.

Theorem 1.2. Assume $(\mathbf{A}(\gamma, \nu))$ for some $\nu \in(0,1)$ and $\gamma \in(0,1]$. Assume also that $b(\theta)=$ $\tilde{b}(\cos \theta)$, for some nondecreasing convex $C^{1}$ function $\tilde{b}$ on $[0,1)$. Let $f_{0}$ be a probability measure on $\mathbb{R}^{2}$ such that for some $\delta \in(\gamma, 2), \int_{\mathbb{R}^{2}} e^{|v|^{\delta}} f_{0}(d v)<\infty$. There exists a unique weak solution $\left(f_{t}\right)_{t \geq 0}$ to (1.1) starting from $f_{0}$. Furthermore, for all $\kappa \in(0, \delta), \sup _{t \geq 0} \int_{\mathbb{R}^{2}} e^{|v|^{\kappa}} f_{t}(d v)<\infty$.

The additional condition that $\tilde{b}$ is nondecreasing and convex is made for convenience, and typically holds if $b(\theta) \simeq|\theta|^{-1-\nu}$.

Sobolev spaces. For $f$ a probability measure on $\mathbb{R}^{2}$, we set, for $\xi \in \mathbb{R}^{2}, \widehat{f}(\xi)=\int_{\mathbb{R}^{2}} e^{i\langle\xi, x\rangle} f(d x)$. Recall that for $r \in \mathbb{R}$,

$$
H^{r}\left(\mathbb{R}^{2}\right)=\left\{f,\|f\|_{H^{r}\left(\mathbb{R}^{2}\right)}<\infty\right\}, \quad \text { where } \quad\|f\|_{H^{r}\left(\mathbb{R}^{2}\right)}^{2}=\int_{\mathbb{R}^{2}}\left(1+|\xi|^{2}\right)^{r}|\widehat{f}(\xi)|^{2} d \xi .
$$

Let us recall the following classical results. For $f$ a probability measure on $\mathbb{R}^{2}$,

- $f \in H^{r}\left(\mathbb{R}^{2}\right)$ for every $r<-1$;

- if $f \in H^{r}\left(\mathbb{R}^{2}\right)$ for some $r \geq 0$, then $f$ has a density that belongs to $L^{2}\left(\mathbb{R}^{2}\right)$;

- if $f \in H^{r}\left(\mathbb{R}^{2}\right)$ for some $r>1$, then $f$ has a bounded and continuous density.

Main result. We need to introduce, for $\nu \in(0,1 / 2)$ and $\gamma \in(0,1)$ satisfying $\gamma>\nu^{2} /(1-2 \nu)$,

$$
\begin{aligned}
& a_{\gamma, \nu}=\frac{1}{2}\left[\sqrt{(\gamma+\nu+1)^{2}+4\left(\frac{\gamma(1-2 \nu)}{\nu}-\nu\right)}-(\gamma+\nu+1)\right]>0, \\
& q_{\gamma, \nu}=\left\{\begin{array}{lll}
a_{\gamma, \nu} & \text { if } \quad a_{\gamma, \nu} \leq 2, \\
\frac{(2+\gamma)(1-2 \nu)-\nu^{2}}{(1+\gamma+\nu) \nu+1} & \text { if } \quad a_{\gamma, \nu}>2 .
\end{array}\right.
\end{aligned}
$$

As we will see in Lemma [5.3 $q_{\gamma, \nu}>2$ in the latter case. 
Theorem 1.3. Assume $(\mathbf{A}(\gamma, \nu))$, for some $\gamma \in(0,1), \nu \in(0,1 / 2)$, such that $\gamma>\nu^{2} /(1-2 \nu)$. Consider a weak solution $\left(f_{t}\right)_{t \in[0, T]}$ to (1.1) such that $f_{0}$ is not a Dirac mass and, for some $\delta \in(\gamma \vee \nu, 1)$

$$
\sup _{t \in[0, T]} \int_{\mathbb{R}^{2}} e^{|v|^{\delta}} f_{t}(d v)<\infty
$$

(i) For all $t_{0} \in(0, T]$,

$$
\begin{aligned}
& \forall q \in\left(0, q_{\gamma, \nu}\right), \quad \forall \xi \in \mathbb{R}^{2}, \quad \sup _{\left[t_{0}, T\right]}\left|\widehat{f}_{t}(\xi)\right| \leq C_{t_{0}, T, q}(1+|\xi|)^{-q}, \\
& \forall r<q_{\gamma, \nu}-1, \quad \sup _{\left[t_{0}, T\right]}\left\|f_{t}\right\|_{H^{r}\left(\mathbb{R}^{2}\right)}<\infty, \\
& \forall q \in\left(0, q_{\gamma, \nu}\right), \quad \forall v_{0} \in \mathbb{R}^{2}, \quad \forall \epsilon>0, \quad \sup _{\left[t_{0}, T\right]} f_{t}\left(\operatorname{Ball}\left(v_{0}, \epsilon\right)\right) \leq C_{t_{0}, T, q} \epsilon^{q} .
\end{aligned}
$$

(ii) If $\nu \in(0,1 / 3)$ and $\gamma>\left(2 \nu+2 \nu^{2}\right) /(1-3 \nu)$, then $q_{\gamma, \nu}>1$. Thus $f_{t}$ has a density belonging to $L^{2}\left(\mathbb{R}^{2}\right)$ for all $t \in(0, T]$.

(iii) If finally $\nu \in(0,1 / 4)$ and $\gamma>\left(6 \nu+3 \nu^{2}\right) /(1-4 \nu)$, then $q_{\gamma, \nu}>2$. Thus $f_{t}$ has a continuous and bounded density for all $t \in(0, T]$.

Discussion about the result. In the realistic case where $\gamma=(s-5) /(s-1)$ and $\nu=2 /(s-1)$, point (i) applies if $s>7$, point (ii) applies if $s>8+\sqrt{33} \simeq 13.75$, point (iii) applies if $s>$ $13+2 \sqrt{31} \simeq 24.14$

When at least point (ii) applies, this shows in particular that for all $t>0, H\left(f_{t}\right)<\infty$, where the entropy is defined as $H(f):=\int_{\mathbb{R}^{2}} f(v) \log f(v) d v$. This allows us to apply many results concerning regularization (see e.g. Villani [15] or Alexandre-Desvillettes-Villani-Wennberg [1]) or large time behavior (see e.g. Villani [16]) where the finiteness of entropy is required.

Until the middle of the 90's, almost all the works on the Boltzmann equation were assuming Grad's angular cutoff, where the cross section $B$, which physically satisfies $\int_{0}^{\pi / 2} B\left(\left|v-v_{*}\right|, \theta\right) d \theta=\infty$ was replaced by an integrable cross section. As shown in Mouhot-Villani [12, no regularization may occur under Grad's angular cutoff. Intuitively, this comes from the fact that each particle is subjected to finitely (resp. infinitely) many collisions on each time interval in the case with (resp. without) cutoff. See however $[8]$ where it is shown on a simplified model that some regularization might occur under Grad's angular cutoff, but for some very soft potentials (i.e. with $\gamma<-1$ ).

Here we deal with true hard potentials and we thus have to overcome the three following difficulties: $|w|^{\gamma}$ vanishes at 0 , explodes at infinity and is not smooth at 0 . This lack of regularity is the basis of many technical complications.

Many papers deal with the case of regularized hard potentials, where $\left|v-v_{*}\right|^{\gamma}$ is replaced by something like $\left(\epsilon^{2}+\left|v-v_{*}\right|^{2}\right)^{\gamma / 2}$. In this situation, Desvillettes-Wennberg [6] have shown that if $f_{0}$ is a function such that $H\left(f_{0}\right)<\infty$, then $f_{t} \in C^{\infty}$ for all $t>0$ for any $\gamma \in(0,1)$, any $\nu \in(0,2)$, in any dimension.

Another simpler situation is the case of Maxwell molecules, where $\gamma=0$ so that $\left|v-v_{*}\right|^{\gamma}$ is constant. Using a probabilistic approach, Graham-Méléard [1] (for the 1-dimensional case) and [7] (for the 2-dimensional case) proved that if $f_{0}$ is a measure with some moments of all orders and is not a Dirac mass, then $f_{t} \in C^{\infty}$ for all $t>0$. In these works, the finiteness of entropy is not required. 
To our knowledge, the only regularization result that concerns true hard potentials is that of Alexandre-Desvillettes-Villani-Wennberg [1]: in any dimension $d \geq 2$, if $f_{0}$ is a function such that $H\left(f_{0}\right)<\infty$, then any weak solution satisfies $\sqrt{f_{t}} \in H_{\text {loc }}^{\nu / 2}\left(\mathbb{R}^{2}\right)$ for all $t>0$, for any value of $\gamma \in(-d, 1)$ and any value of $\nu \in(0,2)$.

Let us compare our result with that of [1. The main limitation of our study is that we work in dimension 2. Furthermore, the result of [1] applies to all potentials, while we have to assume at least $s>7$.

A first positive point is that we assume much less regularity on the initial condition (in [1, $f_{0}$ is already a function): we only assume that $f_{0}$ is not a Dirac mass. This is a necessary condition for regularization, since Dirac masses are stationnary solutions to (1.1).

A second positive point is that we deal with the regularity of $f_{t}$, which seems more natural and tractable than that of $\sqrt{f_{t}}$.

Finally, if $\nu>0$ is small and $\gamma \in(0,1)$ is large, our result seems really competitive. For example if $\gamma=(s-5) /(s-1)$ and $\nu=2 /(s-1)$, then (denoting by $\left.H^{r-}=\cap_{s \in(0, r)} H^{s}\right)$,

- with $s=15$ we obtain $f_{t} \in H^{(1 / 7)-}\left(\mathbb{R}^{2}\right)$ while 1 yields $\sqrt{f_{t}} \in H_{l o c}^{1 / 7}\left(\mathbb{R}^{2}\right)$,

- with $s=25$ we obtain get $f_{t} \in H^{(172 / 167)-}\left(\mathbb{R}^{2}\right)$ while [1] yields $\sqrt{f_{t}} \in H_{\text {loc }}^{1 / 12}\left(\mathbb{R}^{2}\right)$,

- with $s=101$, we obtain $f_{t} \in H^{(4504 / 2599)-}\left(\mathbb{R}^{2}\right)$ while [1] yields $\sqrt{f_{t}} \in H_{\text {loc }}^{1 / 50}\left(\mathbb{R}^{2}\right)$.

Let us finally mention that for any values of $\gamma \in(0,1)$ and $\nu \in(0,1 / 2)$, our result will never provide a better estimate than $f_{t} \in H^{2-}\left(\mathbb{R}^{2}\right)$.

Thus the result of [1] and Theorem 1.3 are complementary: Theorem 1.3 works well for large values of $s$, while [1] works well for small values of $s$. For intermediate values of $s$, Theorem 1.3 allows us to apply [1, even if the initial condition has an infinite entropy.

We conclude this subsection with a remark on regularized hard potentials: if $\nu \in(0,1 / 3)$, our method allows us to extend the result of Desvillettes-Wennberg [6] to initial conditions with infinite entropy.

Remark 1.4. Assume that $B\left(\left|v-v_{*}\right|, \theta\right)=\left(\epsilon^{2}+\left|v-v_{*}\right|^{2}\right)^{\gamma / 2} b(\theta)$, for some $\epsilon>0$, some $\gamma \in(0,1)$ and some $b$ satisfying the same conditions as in $(\mathbf{A}(\gamma, \nu))$ for some $\nu \in(0,1 / 2)$. With our method, it is possible to prove that for $\left(f_{t}\right)_{t \in[0, T]}$ a weak solution to (1.1) satisfying (1.7) and such that $f_{0}$ is not a Dirac mass, for $0<t_{0}<T$, $\sup _{\left[t_{0}, T\right]}\left|\hat{f}_{t}(\xi)\right| \leq C_{t_{0}, T, r}(1+|\xi|)^{-r}$ for all $r \in(0,1 / \nu-2)$. In particular if $\nu \in(0,1 / 3)$, we deduce that $f_{t} \in L^{2}\left(\mathbb{R}^{2}\right)$ so that $H\left(f_{t}\right)<\infty$ for any $t>0$. Thus we can apply the result of [6], and deduce that $f_{t} \in C^{\infty}\left(\mathbb{R}^{2}\right)$ for all $t>0$.

Discussion about the method. Following the seminal work of Tanaka [13, we will build a stochastic process $\left(V_{t}\right)_{t \in[0, T]}$ such that for each $t \in[0, T], \mathcal{L}\left(V_{t}\right)=f_{t}$. This process will solve a jumping stochastic differential equation. Then we will use some Malliavin calculus to study the smoothness of $f_{t}$, in the spirit of Graham-Méléard [11. When using the classical Malliavin calculus for jumps processes of Bichteler-Gravereaux-Jacod [4, one can only treat the case of a constant rate of jump, which corresponds here to the case where $\gamma=0$. This was done in [11, 7]. We thus have to build a suitable Malliavin calculus.

Recently Bally-Clément [2] introduced a new method, still inspired by [4 which allows one to deal with equations with a non-constant rate of jump. They discuss equations with a similar structure as (1.1), but with much more regular coefficients. Here we use the same method, but we have to overcome some nontrivial difficulties related to the singularity and unboundedness of the coefficients. The nondegeneracy property is also quite complicated to establish, in particular because $\left|v-v_{*}\right|^{\gamma}$ vanishes on the diagonal, and because (1.1) is nonlinear. 
Plan of the paper. In the next section, we give the probabilistic interpretation of (1.1) in terms of a jumping S.D.E. We also build some approximations of the process and study their rate of convergence. Another representation of the approximating processes is given in Section 3. In Section 4 we prove an integration by parts formula for the approximating process, using the Malliavin calculus introduced in [2]. We conclude the proof in Section 5] An appendix containing technical results lies at the end of the paper.

Notation. In the whole paper, we assume without loss of generality that

$$
\int_{\mathbb{R}^{2}} v f_{0}(d v)=0 \quad \text { and } \quad e_{0}=\int_{\mathbb{R}^{2}}|v|^{2} f_{0}(d v)>0 .
$$

Observe that $e_{0}>0$, because else, $f_{0}$ would be the Dirac mass at 0 . We always assume at least that $(\mathbf{A}(\gamma, \nu))$ hold for some $\gamma \in(0,1)$, some $\nu \in(0,1)$. We denote by $\left(f_{t}\right)_{t \geq 0}$ a weak solution to (1.1) satisfying (1.7) for some $\delta>\gamma$. We consider $\eta_{0}$ such that

$$
\eta_{0} \in(1 / \delta, 1 /(\gamma \vee \nu)) \text {. }
$$

For $v_{0} \in \mathbb{R}^{2}$ and $r>0$, we denote by

$$
\operatorname{Ball}\left(v_{0}, r\right)=\left\{v \in \mathbb{R}^{2},\left|v-v_{0}\right|<r\right\}
$$

the open ball centered at $v_{0}$ with radius $r$. We will always write $C$ for a finite (large) constant and $c$ for a positive (small) constant, of which the values may change from line to line and which depend only on $b, \nu, \gamma, \delta, \eta_{0}, T, f_{0}$. When a constant depends on another quantity, we will always indicate it. For example, $C_{t_{0}}$ or $c_{t_{0}}$ stand for constants depending on $b, \nu, \gamma, \delta, \eta_{0}, T, f_{0}$ and $t_{0}$.

\section{Probabilistic Interpretation AND APPROXimation}

We first build a Markov process $\left(V_{t}\right)_{t \in[0, T]}$, solution to a jumping stochastic differential equation, whose time marginals will be $\left(f_{t}\right)_{t \in[0, T]}$. The weak solution $\left(f_{t}\right)_{t \in[0, T]}$ being given, we consider a Poisson measure $N(d s, d \theta, d v, d u)$ on $[0, T] \times[-\pi / 2, \pi / 2] \times \mathbb{R}^{2} \times[0, \infty)$ with intensity measure $d s b(\theta) d \theta f_{s}(d v) d u$. Then for a $\mathbb{R}^{2}$-valued $f_{0}$-distributed random variable $V_{0}$ independent of $N$, we consider the $\mathbb{R}^{2}$-valued stochastic differential equation, setting $E=[-\pi / 2, \pi / 2] \times \mathbb{R}^{2} \times[0, \infty)$,

$$
V_{t}=V_{0}+\int_{0}^{t} \int_{E} A(\theta)\left(V_{s-}-v\right) \mathbb{I}_{\left\{u \leq\left|V_{s-}-v\right|^{\gamma}\right\}} N(d s, d \theta, d v, d u) .
$$

We also introduce some approximations of the process $\left(V_{t}\right)_{t \in[0, T]}$. We consider a $C^{\infty}$ even nonnegative function $\chi$ supported by $(-1,1)$ satisfying $\int_{\mathbb{R}} \chi(x) d x=1$. Then we introduce, for $x \in \mathbb{R}$ and $\epsilon \in(0,1)$, (recall (1.9) $)$

$$
\Gamma_{\epsilon}=[\log (1 / \epsilon)]^{\eta_{0}}, \quad \phi_{\epsilon}(x)=\int_{\mathbb{R}}\left((y \vee 2 \epsilon) \wedge \Gamma_{\epsilon}\right) \frac{\chi((x-y) / \epsilon)}{\epsilon} d y .
$$

Observe that we have $2 \epsilon \leq \phi_{\epsilon}(x) \leq \Gamma_{\epsilon}$ for all $x \geq 0, \phi_{\epsilon}(x)=x$ for $x \in\left[3 \epsilon, \Gamma_{\epsilon}-1\right], \phi_{\epsilon}(x)=2 \epsilon$ for $x \in[0, \epsilon]$ and $\phi_{\epsilon}(x)=\Gamma_{\epsilon}$ for $x \geq \Gamma_{\epsilon}+1$. We find $\epsilon_{0}>0$ small enough, in such a way that for $\epsilon \in\left(0, \epsilon_{0}\right), 3 \epsilon<1<\Gamma_{\epsilon}-1$ and consider, for $\epsilon \in\left(0, \epsilon_{0}\right)$, the equation

$$
V_{t}^{\epsilon}=V_{0}+\int_{0}^{t} \int_{E} A(\theta)\left(V_{s-}^{\epsilon}-v\right) \mathbb{1}_{\left\{u \leq \phi_{\epsilon}^{\gamma}\left(\left|V_{s-}^{\epsilon}-v\right|\right)\right\}} N(d s, d \theta, d v, d u),
$$


Next we introduce, for $\zeta \in(0,1)$, a function $I_{\zeta}: \mathbb{R}_{+} \mapsto[0,1]$ such that $I_{\zeta}(x)=1$ for $x \geq \zeta$ and vanishing on a neighborhood of 0 . We will choose $I_{\zeta}$ in the next section as a smooth version of $\mathbb{I}_{\{x \geq \zeta\}}$. We consider the equation

$$
V_{t}^{\epsilon, \zeta}=V_{0}+\int_{0}^{t} \int_{E} A(\theta)\left(V_{s-}^{\epsilon, \zeta}-v\right) \mathbb{I}_{\left\{u \leq \phi_{\epsilon}^{\gamma}\left(\left|V_{s-}^{\epsilon, \zeta}-v\right|\right)\right\}} I_{\zeta}(|\theta|) N(d s, d \theta, d v, d u) .
$$

The goal of this section is to check the following results.

Proposition 2.1. (i) There exists a unique càdlàg adapted solution $\left(V_{t}\right)_{t \in[0, T]}$ to (2.1). For each $\epsilon \in\left(0, \epsilon_{0}\right)$ and each $\zeta \in(0,1)$, there exist some unique càdlàg adapted solutions $\left(V_{t}^{\epsilon}\right)_{t \in[0, T]}$ and $\left(V_{t}^{\epsilon, \zeta}\right)_{t \in[0, T]}$ to (2.3) and 2.4).

(ii) For all $t \in[0, T], V_{t}$ is $f_{t}$-distributed.

(iii) For any $\kappa \in(\nu, \delta)$, any $\epsilon \in\left(0, \epsilon_{0}\right)$, any $\zeta \in(0,1)$,

$$
\mathbb{E}\left[\sup _{[0, T]}\left(e^{\left|V_{t}\right|^{\kappa}}+e^{\left|V_{t}^{\epsilon}\right|^{\kappa}}+e^{\left|V_{t}^{\epsilon, \zeta}\right|^{\kappa}}\right)\right] \leq C_{\kappa} .
$$

(iv) For any $\beta \in(\nu, 1]$, any $\epsilon \in\left(0, \epsilon_{0}\right)$, any $\zeta \in(0,1)$,

$$
\sup _{[0, T]} \mathbb{E}\left[\left|V_{t}^{\epsilon}-V_{t}^{\epsilon, \zeta}\right|^{\beta}\right] \leq C_{\beta} e^{C_{\beta} \Gamma_{\epsilon}^{\gamma}} \zeta^{\beta-\nu} .
$$

(v) Assume furthermore that for some $\alpha \geq 0$, some $K$, for all $v_{0} \in \mathbb{R}^{2}$, for all $\epsilon \in(0,1]$,

$$
\sup _{[0, T]} f_{t}\left(\operatorname{Ball}\left(v_{0}, \epsilon\right)\right) \leq K \epsilon^{\alpha} .
$$

This always holds with $K=1, \alpha=0$. Then for any $\beta \in(\nu, 1]$, any $\epsilon \in\left(0, \epsilon_{0}\right)$, any $\zeta \in(0,1)$,

$$
\sup _{[0, T]} \mathbb{E}\left[\left|V_{t}-V_{t}^{\epsilon}\right|^{\beta}\right] \leq C_{\beta, K} e^{C_{\beta} \Gamma_{\epsilon}^{\gamma}} \epsilon^{\beta+\gamma+\alpha} .
$$

Observe that $e^{C \Gamma_{\epsilon}^{\gamma}}$ is not very large: since $\Gamma_{\epsilon}^{\gamma}=[\log (1 / \epsilon)]^{\gamma \eta_{0}}$ with $\gamma \eta_{0}<1$ (recall (1.9)), we have $e^{C \Gamma_{\epsilon}^{\gamma}} \leq C_{\eta} \epsilon^{-\eta}$, for any $\eta>0$.

Proof. We handle the proof in several steps. In Steps 1-5, we assume that $\left(V_{t}\right)_{t \in[0, T]},\left(V_{t}^{\epsilon}\right)_{t \in[0, T]}$ and $\left(V_{t}^{\epsilon, \zeta}\right)_{t \in[0, T]}$ exist and prove points (iii)-(v). Points (i) and (ii) are then checked in Steps 6-7.

Step 1. We first check that for $\kappa \in(\nu, \delta)$,

$$
\sup _{[0, T]} \mathbb{E}\left[e^{\left|V_{t}\right|^{\kappa}}+e^{\left|V_{t}^{\epsilon}\right|^{\kappa}}+e^{\left|V_{t}^{\epsilon, \zeta}\right|^{\kappa}}\right] \leq C_{\kappa} .
$$

Let us for example treat the case of $\left(V_{t}^{\epsilon}\right)_{t \in[0, T]}$. We have

$$
e^{\left|V_{t}^{\epsilon}\right|^{\kappa}}=e^{\left|V_{0}\right|^{\kappa}}+\int_{0}^{t} \int_{E}\left[e^{\left|V_{s-}^{\epsilon}+A(\theta)\left(V_{s-}^{\epsilon}-v\right)\right|^{\kappa}}-e^{\left|V_{s-}^{\epsilon}\right|^{\kappa}}\right] \mathbb{I}_{\left\{u \leq \phi_{\epsilon}^{\gamma}\left(\left|V_{s-}^{\epsilon}-v\right|\right)\right\}} N(d s, d \theta, d v, d u) .
$$


Taking expectations and using Lemma 6.4.

$$
\begin{aligned}
& \mathbb{E}\left[e^{\left|V_{t}^{\epsilon}\right|^{\kappa}}\right]= \mathbb{E}\left[e^{\left|V_{0}\right|^{\kappa}}\right]+\int_{0}^{t} d s \int_{-\pi / 2}^{\pi / 2} b(\theta) d \theta \int_{\mathbb{R}^{2}} f_{s}(d v) \\
& \mathbb{E}\left[\left(e^{\left|V_{s}^{\epsilon}+A(\theta)\left(V_{s}^{\epsilon}-v\right)\right|^{\kappa}}-e^{\left|V_{s}^{\epsilon}\right|^{\kappa}}\right) \phi_{\epsilon}^{\gamma}\left(\left|V_{s}^{\epsilon}-v\right|\right)\right] \\
& \leq \mathbb{E}\left[e^{\left|V_{0}\right|^{\kappa}}\right]+\int_{0}^{t} d s \int_{\mathbb{R}^{2}} f_{s}(d v) \mathbb{E}\left[\phi_{\epsilon}^{\gamma}\left(\left|V_{s}^{\epsilon}-v\right|\right) e^{\left|V_{s}^{\epsilon}\right|^{\kappa}}\right. \\
&\left.\quad\left(-c_{\kappa} \mathbb{I}_{\left\{\left|V_{s}^{\epsilon}\right| \geq 1,\left|V_{s}^{\epsilon}\right| \geq C|v|\right\}}+C_{\kappa}\left(\left|V_{s}^{\epsilon}\right| \vee 1\right)^{\kappa+\nu-2} e^{C_{\kappa}|v|^{\kappa}}\right)\right] .
\end{aligned}
$$

But $\kappa+\nu-2<0$, so that for $|V| \geq M_{\kappa}(v):=\max \left\{1, C|v|,\left[C_{\kappa} e^{C_{\kappa}|v|^{\kappa}} / c_{\kappa}\right]^{1 /(2-\nu-\kappa)}\right\}$, we have

$$
-c_{\kappa} \mathbb{I}_{\{|V| \geq 1,|V| \geq C|v|\}}+C_{\kappa}(|V| \vee 1)^{\kappa+\nu-2} e^{C_{\kappa}|v|^{\kappa}} \leq 0 .
$$

Changing the values of the constants, $M_{\kappa}(v) \leq C_{\kappa} e^{C_{\kappa}|v|^{\kappa}}$. Thus

$$
\mathbb{E}\left[e^{\left|V_{t}^{\epsilon}\right|^{\kappa}}\right] \leq \mathbb{E}\left[e^{\left|V_{0}\right|^{\kappa}}\right]+C_{\kappa} \int_{0}^{t} d s \int_{\mathbb{R}^{2}} f_{s}(d v) \mathbb{E}\left[\phi_{\epsilon}^{\gamma}\left(\left|V_{s}^{\epsilon}-v\right|\right) e^{\left|V_{s}^{\epsilon}\right|^{\kappa}} \mathbb{I}_{\left\{\left|V_{s}^{\epsilon}\right| \leq C_{\kappa} e^{\left.C_{\kappa}|v|^{\kappa}\right\}}\right.} e^{C_{\kappa}|v|^{\kappa}}\right] .
$$

Since now $\phi_{\epsilon}^{\gamma}(|V-v|) \leq(1+|V|+|v|)^{\gamma}$, we deduce that $\phi_{\epsilon}^{\gamma}(|V-v|) \mathbb{1}_{\left\{|V| \leq C_{\kappa} e^{\left.C_{\kappa}|v|^{\kappa}\right\}}\right.} e^{C_{\kappa}|v|^{\kappa}} \leq$ $C_{\kappa} e^{C_{\kappa}|v|^{\kappa}}$, whence

$$
\mathbb{E}\left[e^{\left|V_{t}^{\epsilon}\right|^{\kappa}}\right] \leq \mathbb{E}\left[e^{\left|V_{0}\right|^{\kappa}}\right]+C_{\kappa} \int_{0}^{t} d s \int_{\mathbb{R}^{2}} f_{s}(d v) \mathbb{E}\left[e^{\left|V_{s}^{\epsilon}\right|^{\kappa}}\right] e^{C_{\kappa}|v|^{\kappa}} \leq C_{\kappa}+C_{\kappa} \int_{0}^{t} d s \mathbb{E}\left[e^{\left|V_{s}^{\epsilon}\right|^{\kappa}}\right] .
$$

We finally used (1.7), that $\kappa<\delta$ and that $V_{0} \sim f_{0}$. The Gronwall Lemma allows us to conclude.

Step 2. We now prove (iii), for example with $\left(V_{t}^{\epsilon}\right)_{t \in[0, T]}$. Using (2.5) and Lemma 6.4, we obtain

$$
\begin{aligned}
& \mathbb{E}\left[\sup _{[0, T]} e^{\left|V_{t}^{\epsilon}\right|^{\kappa}}\right] \leq \mathbb{E}\left[e^{\left|V_{0}\right|^{\kappa}}\right]+\int_{0}^{T} d s \int_{-\pi / 2}^{\pi / 2} b(\theta) d \theta \int_{\mathbb{R}^{2}} f_{s}(d v) \\
& \mathbb{E}\left[\left|e^{\left|V_{s}^{\epsilon}+A(\theta)\left(V_{s}^{\epsilon}-v\right)\right|^{\kappa}}-e^{\left|V_{s}^{\epsilon}\right|^{\kappa}}\right| \phi_{\epsilon}^{\gamma}\left(\left|V_{s}^{\epsilon}-v\right|\right)\right] \\
& \leq C_{\kappa}+C_{\kappa} \int_{0}^{T} d s \int_{\mathbb{R}^{2}} f_{s}(d v) \mathbb{E}\left[\phi_{\epsilon}^{\gamma}\left(\left|V_{s}^{\epsilon}-v\right|\right) e^{C_{\kappa}|v|^{\kappa}} e^{C_{\kappa}\left|V_{s}^{\epsilon}\right|^{\kappa}}\right] \\
& \leq C_{\kappa}+C_{\kappa} \int_{0}^{T} d s \int_{\mathbb{R}^{2}} f_{s}(d v) e^{C_{\kappa}|v|^{\kappa}} \mathbb{E}\left[e^{C_{\kappa}\left|V_{s}^{\epsilon}\right|^{\kappa}}\right] .
\end{aligned}
$$

We used here that $\phi_{\epsilon}^{\gamma}(|V-v|) e^{C_{\kappa}|V|^{\kappa}} e^{C_{\kappa}|v|^{\kappa}} \leq(1+|V|+|v|)^{\gamma} e^{C_{\kappa}|V|^{\kappa}} e^{C_{\kappa}|v|^{\kappa}} \leq e^{C_{\kappa}|V|^{\kappa}} e^{C_{\kappa}|v|^{\kappa}}$. Step 1 and (1.7) allow us to conclude, for $\kappa \in(\nu, \delta)$.

Step 3. We set

$$
h(u, v, \theta, w)=A(\theta)(w-v) \mathbb{1}_{\left\{u \leq|w-v|^{\gamma}\right\}} \text { and } h_{\epsilon}(u, v, \theta, w)=A(\theta)(w-v) \mathbb{I}_{\left\{u \leq \phi_{\epsilon}^{\gamma}(|w-v|)\right\}}
$$

and we prove that for $\beta \in(0,1]$,

$$
\begin{aligned}
& \int_{0}^{\infty}\left|\left(h-h_{\epsilon}\right)(u, v, \theta, w)\right|^{\beta} d u \leq C|\theta|^{\beta}|w-v|^{\beta}\left(\epsilon^{\gamma} \mathbb{1}_{\{|w-v| \leq 3 \epsilon\}}+|w-v|^{\gamma} \mathbb{I}_{\left\{|w-v| \geq \Gamma_{\epsilon}-1\right\}}\right), \\
& \int_{0}^{\infty}\left|h_{\epsilon}(u, v, \theta, w)-h_{\epsilon}(u, v, \theta, \tilde{w})\right|^{\beta} d u \leq C_{\beta}|\theta|^{\beta} \Gamma_{\epsilon}^{\gamma}|w-\tilde{w}|^{\beta}
\end{aligned}
$$


We notice that $|A(\theta)| \leq|\theta|$ (see (1.2)) and recall that $\phi_{\epsilon}(x)=x$ for $x \in\left[3 \epsilon, \Gamma_{\epsilon}-1\right]$, that $\phi_{\epsilon}(x) \leq 3 \epsilon$ for $x \in[0,3 \epsilon]$ and that $\phi_{\epsilon}(x) \leq x$ for $x \geq \Gamma_{\epsilon}-1$. The left hand side of (2.6) is bounded by

$$
\begin{aligned}
& |\theta|^{\beta}|w-v|^{\beta} \int_{0}^{\infty}\left|\mathbb{1}_{\left\{u \leq|v-w|^{\gamma}\right\}}-\mathbb{1}_{\left\{u \leq \phi_{\epsilon}^{\gamma}(|v-w|)\right.}\right| d u \\
\leq & |\theta|^{\beta}|w-v|^{\beta}|| v-\left.w\right|^{\gamma}-\phi_{\epsilon}^{\gamma}(|v-w|) \mid \\
\leq & |\theta|^{\beta}|w-v|^{\beta}\left(\mathbb{I}_{\{|w-v| \leq 3 \epsilon\}}+\mathbb{I}_{\left\{|w-v| \geq \Gamma_{\epsilon}-1\right\}}\right)|| w-\left.v\right|^{\gamma}-\phi_{\epsilon}^{\gamma}(|w-v|) \mid \\
\leq & |\theta|^{\beta}|w-v|^{\beta}\left(\mathbb{I}_{\{|w-v| \leq 3 \epsilon\}}(3 \epsilon)^{\gamma}+\mathbb{I}_{\left\{|w-v| \geq \Gamma_{\epsilon}-1\right\}}|w-v|^{\gamma}\right) .
\end{aligned}
$$

Similarly, using Lemma 6.3.(i) and that $\phi_{\epsilon} \leq \Gamma_{\epsilon}$, the left hand side of (2.7) is bounded by

$$
\begin{aligned}
& |\theta|^{\beta}|(w-v)-(\tilde{w}-v)|^{\beta} \phi_{\epsilon}^{\gamma}(|w-v|)+|\theta|^{\beta}|\tilde{w}-v|^{\beta}\left|\phi_{\epsilon}^{\gamma}(|w-v|)-\phi_{\epsilon}^{\gamma}(|\tilde{w}-v|)\right| \\
\leq & |\theta|^{\beta}|w-\tilde{w}|^{\beta} \Gamma_{\epsilon}^{\gamma}+C_{\beta}|\theta|^{\beta} \Gamma_{\epsilon}^{\gamma}|| w-v|-| \tilde{w}-v||^{\beta} \leq C_{\beta}|\theta|^{\beta}|w-\tilde{w}|^{\beta} \Gamma_{\epsilon}^{\gamma} .
\end{aligned}
$$

Step 4. We now prove (iv). Let thus $\beta \in(\nu, 1]$. Since $x \mapsto x^{\beta}$ is sub-additive, we can write

$$
\begin{aligned}
\mathbb{E}\left[\left|V_{t}^{\epsilon}-V_{t}^{\epsilon, \zeta}\right|^{\beta}\right] \leq & \int_{0}^{t} d s \int_{-\pi / 2}^{\pi / 2} b(\theta) d \theta \int_{\mathbb{R}^{2}} f_{s}(d v) \int_{0}^{\infty} d u \mathbb{E}\left[\left|h_{\epsilon}\left(u, v, \theta, V_{s}^{\epsilon, \zeta}\right)-h_{\epsilon}\left(u, v, \theta, V_{s}^{\epsilon}\right)\right|^{\beta}\right] \\
& +\int_{0}^{t} d s \int_{-\pi / 2}^{\pi / 2}\left(1-I_{\zeta}(|\theta|)\right)^{\beta} b(\theta) d \theta \int_{\mathbb{R}^{2}} f_{s}(d v) \int_{0}^{\infty} d u \mathbb{E}\left[\left|h_{\epsilon}\left(u, v, \theta, V_{s}^{\epsilon, \zeta}\right)\right|^{\beta}\right] .
\end{aligned}
$$

Using (2.7) and that $0 \leq 1-I_{\zeta}(|\theta|) \leq \mathbb{1}_{\{|\theta| \leq \zeta\}}$, we get

$$
\begin{aligned}
\mathbb{E}\left[\left|V_{t}^{\epsilon}-V_{t}^{\epsilon, \zeta}\right|^{\beta}\right] \leq & C_{\beta} \Gamma_{\epsilon}^{\gamma} \int_{0}^{t} d s \int_{-\pi / 2}^{\pi / 2} b(\theta) d \theta|\theta|^{\beta} \mathbb{E}\left[\left|V_{s}^{\epsilon}-V_{s}^{\epsilon, \zeta}\right|^{\beta}\right] \\
& +C_{\beta} \int_{0}^{t} d s \int_{-\zeta}^{\zeta} b(\theta) d \theta|\theta|^{\beta} \int_{\mathbb{R}^{2}} f_{s}(d v) \mathbb{E}\left[\phi_{\epsilon}^{\gamma}\left(\left|V_{s}^{\epsilon, \zeta}-v\right|\right)\left|V_{s}^{\epsilon, \zeta}-v\right|^{\beta}\right] .
\end{aligned}
$$

Using $(\mathbf{A}(\gamma, \nu))$, since $\beta>\nu$ and since $\phi_{\epsilon}^{\gamma}(|V-v|)|V-v|^{\beta} \leq C\left(1+|v|^{2}+|V|^{2}\right)$, this yields

$$
\begin{aligned}
\mathbb{E}\left[\left|V_{t}^{\epsilon}-V_{t}^{\epsilon, \zeta}\right|^{\beta}\right] \leq & C_{\beta} \Gamma_{\epsilon}^{\gamma} \int_{0}^{t} \mathbb{E}\left[\left|V_{s}^{\epsilon}-V_{s}^{\epsilon, \zeta}\right|^{\beta}\right] d s \\
& +C_{\beta} \zeta^{\beta-\nu} \int_{0}^{t} d s \int_{\mathbb{R}^{2}} f_{s}(d v) \mathbb{E}\left[1+\left|V_{s}^{\epsilon, \zeta}\right|^{2}+|v|^{2}\right] \\
\leq & C_{\beta} \Gamma_{\epsilon}^{\gamma} \int_{0}^{t} \mathbb{E}\left[\left|V_{s}^{\epsilon}-V_{s}^{\epsilon, \zeta}\right|^{\beta}\right] d s+C_{\beta} \zeta^{\beta-\nu}
\end{aligned}
$$

where we used (1.7) and point (iii). The Gronwall Lemma allows us to conclude.

Step 5. Let us check (v), for some $\beta \in(\nu, 1]$ fixed. Using again the sub-additivity of $x \mapsto x^{\beta}$, (2.6] 2.7), $(\mathbf{A}(\gamma, \nu))$ and that $\beta>\nu$, we obtain

$$
\mathbb{E}\left[\left|V_{t}-V_{t}^{\epsilon}\right|^{\beta}\right] \leq \int_{0}^{t} d s \int_{-\pi / 2}^{\pi / 2} b(\theta) d \theta \int_{\mathbb{R}^{2}} f_{s}(d v) \int_{0}^{\infty} d u \mathbb{E}\left[\left|h\left(u, v, \theta, V_{s}\right)-h_{\epsilon}\left(u, v, \theta, V_{s}^{\epsilon}\right)\right|^{\beta}\right] .
$$


We infer from (2.6][2.7), $(\mathbf{A}(\gamma, \nu))$ and the fact that $\beta>\nu$ that

$$
\begin{aligned}
& \mathbb{E}\left[\left|V_{t}-V_{t}^{\epsilon}\right|^{\beta}\right] \leq C_{\beta} \int_{0}^{t} d s \int_{-\pi / 2}^{\pi / 2} b(\theta) d \theta|\theta|^{\beta} \int_{\mathbb{R}^{2}} f_{s}(d v) \\
& \mathbb{E}\left(\left|V_{s}-v\right|^{\beta}\left(\epsilon^{\gamma} \mathbb{I}_{\left\{\left|V_{s}-v\right| \leq 3 \epsilon\right\}}+\left|V_{s}-v\right|^{\gamma} \mathbb{I}_{\left\{\left|V_{s}-v\right| \geq \Gamma_{\epsilon}-1\right\}}\right)+\Gamma_{\epsilon}^{\gamma}\left|V_{s}-V_{s}^{\epsilon}\right|^{\beta}\right) \\
& \leq C_{\beta} \epsilon^{\beta+\gamma} \int_{0}^{t} d s \mathbb{E}\left[f_{s}\left(\operatorname{Ball}\left(V_{s}, 3 \epsilon\right)\right)\right]+C_{\beta} \Gamma_{\epsilon}^{\gamma} \int_{0}^{t} d s \mathbb{E}\left[\left|V_{s}-V_{s}^{\epsilon}\right|^{\beta}\right] \\
&\left.+C_{\beta} \int_{0}^{t} d s \int_{\mathbb{R}^{2}} f_{s}(d v) \mathbb{E}\left[\left|V_{s}-v\right|^{\beta+\gamma} \mathbb{I}_{\left\{\left|V_{s}-v\right| \geq \Gamma_{\epsilon}-1\right\}}\right)\right] .
\end{aligned}
$$

By assumption, we have

$$
\sup _{[0, T]} \mathbb{E}\left[f_{s}\left(\operatorname{Ball}\left(V_{s}, 3 \epsilon\right)\right)\right] \leq 3^{\alpha} K \epsilon^{\alpha} .
$$

Next (1.7) and point (iii) yield, for $\kappa \in\left(1 / \eta_{0}, \delta\right)$,

$$
\begin{aligned}
\int_{\mathbb{R}^{2}} f_{s}(d v) \mathbb{E}\left[\left|V_{s}-v\right|^{\beta+\gamma} \mathbb{I}_{\left.\left\{\left|V_{s}-v\right| \geq \Gamma_{\epsilon}-1\right\}\right)}\right] & \left.\leq \int_{\mathbb{R}^{2}} f_{s}(d v) \mathbb{E}\left[\left(\left|V_{s}\right|+|v|\right)^{\beta+\gamma} \mathbb{I}_{\left\{\left|V_{s}\right|+|v| \geq \Gamma_{\epsilon}-1\right\}}\right)\right] \\
& \leq e^{-\left(\Gamma_{\epsilon}-1\right)^{\kappa}} \int_{\mathbb{R}^{2}} f_{s}(d v) \mathbb{E}\left[\left(\left|V_{s}\right|+|v|\right)^{\beta+\gamma} e^{\left(\left|V_{s}\right|+|v|\right)^{\kappa}}\right] \\
& \leq C_{\kappa} e^{-\Gamma_{\epsilon}^{\kappa}} \int_{\mathbb{R}^{2}} f_{s}(d v) \mathbb{E}\left[e^{C_{\kappa}\left(\left|V_{s}\right|+|v|\right)^{\kappa}}\right] \leq C_{\kappa} e^{-\Gamma_{\epsilon}^{\kappa}}
\end{aligned}
$$

Thus we have

$$
\mathbb{E}\left[\left|V_{t}-V_{t}^{\epsilon}\right|^{\beta}\right] \leq C_{\beta, \kappa, K}\left(\epsilon^{\beta+\gamma+\alpha}+e^{-\Gamma_{\epsilon}^{\kappa}}\right)+C_{\beta} \Gamma_{\epsilon}^{\gamma} \int_{0}^{t} d s \mathbb{E}\left[\left|V_{s}-V_{s}^{\epsilon}\right|^{\beta}\right],
$$

whence $\mathbb{E}\left[\left|V_{t}-V_{t}^{\epsilon}\right|^{\beta}\right] \leq C_{\beta, \kappa, K}\left(\epsilon^{\beta+\gamma+\alpha}+e^{-\Gamma_{\epsilon}^{\kappa}}\right) e^{C_{\beta} \Gamma_{\epsilon}^{\gamma} T}$ by the Gronwall Lemma. We easily conclude, since $\kappa>\gamma$ and since $\Gamma_{\epsilon}^{\kappa}=[\log (1 / \epsilon)]^{\kappa \eta_{0}}$, with $\kappa \eta_{0}>1$.

Step 6. We now prove point (i). First, the strong existence and uniqueness of a solution $\left(V_{t}^{\epsilon, \zeta}\right)_{t \in[0, T]}$ to (2.4) is obvious, since the Poisson measure used in (2.4) is a.s. finite because since $I_{\zeta}$ vanishes on a neighborhood of 0 ,

$$
\int_{0}^{T} \int_{E} \mathbb{1}_{\left\{I_{\zeta}(|\theta|) \neq 0, u \leq \Gamma_{\epsilon}^{\gamma}\right\}} d s b(\theta) d \theta f_{s}(d v) d u<\infty .
$$

Similar arguments as in point (iv) allow us to pass to the limit as $\zeta \rightarrow 0$ (recall that $I_{\zeta}(|\theta|) \rightarrow$ $\left.\mathbb{1}_{\{\theta \neq 0\}}\right)$ and to deduce that there exists a unique solution to $\left(V_{t}^{\epsilon}\right)_{t \in[0, T]}$ to (2.3). Finally, we use similar arguments as in point $(\mathrm{v})$ to prove the existence and uniqueness of a solution $\left(V_{t}\right)_{t \in[0, T]}$ to (2.1), by taking the limit $\epsilon \rightarrow 0$.

Step \%. It remains to show that $V_{t} \sim f_{t}$ for all $t \in[0, T]$. To this end, we denote by $g_{t}$ the law of $V_{t}$. Then $g_{0}=f_{0}$ by assumption. Using the Itô formula for jump processes and taking expectations, we see that $\left(g_{t}\right)_{t \in[0, T]}$ solves the following linear Boltzmann equation: for all $\psi: \mathbb{R}^{2} \mapsto \mathbb{R}$ globally Lipschitz continuous,

$$
\frac{d}{d t} \int_{\mathbb{R}^{2}} \psi(v) g_{t}(d v)=\int_{\mathbb{R}^{2}} g_{t}(d v) \int_{\mathbb{R}^{2}} f_{t}\left(d v_{*}\right) \int_{-\pi / 2}^{\pi / 2} b(\theta) d \theta\left|v-v_{*}\right|^{\gamma}\left[\psi\left(v+A(\theta)\left(v-v_{*}\right)\right)-\psi(v)\right] .
$$


Of course, $\left(f_{t}\right)_{t \in[0, T]}$ also solves this linear equation. Thus $\left(g_{t}\right)_{t \in[0, T]}=\left(f_{t}\right)_{t \in[0, T]}$ by a uniqueness argument. The uniqueness for this linear equation can be derived from the uniqueness of the solution to (2.1), by using the results of Bhatt-Karandikar [3, Theorem 5.2], see [9, Lemma 4.6] for very similar considerations in a very close situation.

\section{Some Substitutions}

The Malliavin calculus we will use in the next sections concerns the solution $\left(V_{t}^{\epsilon, \zeta}\right)_{t \in[0, T]}$ of (2.4). Since $\phi_{\epsilon}^{\gamma} \leq \Gamma_{\epsilon}^{\gamma} \leq 2 \Gamma_{\epsilon}^{\gamma}$ (we will need a few scope), we can write

$$
V_{t}^{\epsilon, \zeta}=V_{0}+\int_{0}^{t} \int_{-\pi / 2}^{\pi / 2} \int_{\mathbb{R}^{2}} \int_{0}^{2 \Gamma_{\epsilon}^{\gamma}} A(\theta)\left(V_{s-}^{\epsilon, \zeta}-v\right) I_{\zeta}(|\theta|) \mathbb{I}_{\left\{u \leq \phi_{\epsilon}^{\gamma}\left(\left|V_{s-}^{\epsilon, \zeta}-v\right|\right)\right\}} N(d s, d \theta, d v, d u) .
$$

Recall that the instensity measure of $N$ is given by $d s b(\theta) d \theta f_{t}(d v) d u$. Our goal in this section is to modify this formula in order to get an expression in adequacy with 2]. First of all, we use the Skorokhod representation Theorem to find a measurable application $v_{t}:[0,1] \mapsto \mathbb{R}^{2}$ such that for all $\psi: \mathbb{R}^{2} \mapsto \mathbb{R}_{+}$,

$$
\int_{0}^{1} \psi\left(v_{t}(\rho)\right) d \rho=\int_{\mathbb{R}^{2}} \psi(v) f_{t}(d v)
$$

Next, we consider the following function $G: x \in(0, \pi / 2) \mapsto(0, \infty)$

$$
G(x)=\int_{x}^{\pi / 2} b(\theta) d \theta
$$

and its inverse $\vartheta:(0, \infty) \mapsto(0, \pi / 2)$ (i.e. $G(\vartheta(z))=z$ ) and we set $\vartheta(z)=-\vartheta(-z)$ if $z<0$. Then for all $\psi:[-\pi / 2, \pi / 2] \backslash\{0\} \mapsto \mathbb{R}_{+}$,

$$
\int_{-\pi / 2}^{\pi / 2} \psi(\theta) b(\theta) d \theta=\int_{\mathbb{R}_{*}} \psi(\vartheta(z)) d z .
$$

Notice that $\vartheta$ is smooth on $(-\infty, 0) \cup(0, \infty)$. Since $b(\theta) \simeq|\theta|^{-1-\nu}$ by assumption, we have $G(x) \simeq \nu^{-1}\left(x^{-\nu}-(\pi / 2)^{-\nu}\right)$, and thus $\vartheta(z) \simeq\left(\nu z+(2 / \pi)^{\nu}\right)^{-1 / \nu} \simeq(1+z)^{-1 / \nu}$. See Lemma 6.2 for some precise estimates.

Observe now that for all $z \in \mathbb{R}_{*}$,

$$
|\vartheta(z)|>\zeta \Longleftrightarrow|z|<G(\zeta)
$$

We choose $I_{\zeta}$ in such a way that for $\mathbf{I}_{\zeta}(z)=I_{\zeta}(\vartheta(|z|)), \mathbf{I}_{\zeta}: \mathbb{R} \mapsto[0,1]$ is smooth (with all its derivatives bounded uniformly in $\zeta$ ) and verifies $\mathbf{I}_{\zeta}(z)=1$ for $|z| \leq G(\zeta)$ and $\mathbf{I}_{\zeta}(z)=0$ for $|z| \geq G(\zeta)+1$.

We can write, using the substitutions $\theta=\vartheta(z)$ and $v=v_{s}(\rho)$,

$$
V_{t}^{\epsilon, \zeta}=V_{0}+\int_{0}^{t} \int_{0}^{1} \int_{-G(\zeta)-1}^{G(\zeta)+1} \int_{0}^{2 \Gamma_{\epsilon}^{\gamma}} A(\vartheta(z))\left(V_{s-}^{\epsilon, \zeta}-v_{s}(\rho)\right) \mathbf{I}_{\zeta}(z) \mathbb{1}_{\left\{u \leq \phi_{\epsilon}^{\gamma}\left(\left|V_{s-}^{\epsilon, \zeta}-v_{s}(\rho)\right|\right)\right\}} M(d s, d \rho, d z, d u),
$$

where $M$ is a Poisson measure on $[0, T] \times[0,1] \times \mathbb{R}_{*} \times[0, \infty)$ with intensity measure $d s d \rho d z d u$. These subsitutions are used for technical convenience: for example, it would have been technically complicated to use a smooth version of $\mathbb{1}_{\{|\theta| \geq \zeta\}}$ (with $\zeta$ small), while it is easy to build a smooth version of $\mathbb{I}_{\{|z| \geq G(\zeta)\}}$ (with $G(\zeta)$ large), see also Remark 4.2 below. 
Consequently, there exists a standard Poisson process $J_{t}^{\epsilon, \zeta}=\sum_{k \geq 1} \mathbb{I}_{\left\{T_{k}^{\epsilon, \zeta} \leq t\right\}}$ with rate

$$
\lambda_{\epsilon, \zeta}=\int_{0}^{1} d \rho \int_{-G(\zeta)-1}^{G(\zeta)+1} d z \int_{0}^{2 \Gamma_{\epsilon}^{\gamma}} d u=4(G(\zeta)+1) \Gamma_{\epsilon}^{\gamma}
$$

and a family $\left(\bar{R}_{k}^{\epsilon, \zeta}, \bar{Z}_{k}^{\epsilon, \zeta}, \bar{U}_{k}^{\epsilon, \zeta}\right)_{k \geq 1}$ of i.i.d. $[0,1] \times[-G(\zeta)-1, G(\zeta)+1] \times\left[0,2 \Gamma_{\epsilon}^{\gamma}\right]$-valued random variables with law $\lambda_{\epsilon, \zeta}^{-1} d \rho d z d u$ such that, with the conventions $\sum_{1}^{0}=0$ and $T_{0}^{\epsilon, \zeta}=0$,

$$
V_{t}^{\epsilon, \zeta}=V_{0}+\sum_{k=1}^{J_{t}^{\epsilon, \zeta}} A\left(\vartheta\left(\bar{Z}_{k}^{\epsilon, \zeta}\right)\right)\left(V_{T_{k-1}^{\epsilon, \zeta}}^{\epsilon, \zeta}-v_{T_{k}^{\epsilon, \zeta}}\left(\bar{R}_{k}^{\epsilon, \zeta}\right)\right) \mathbf{I}_{\zeta}\left(Z_{k}^{\epsilon, \zeta}\right) \mathbb{I}_{\left\{\bar{U}_{k}^{\epsilon, \zeta} \leq \phi_{\epsilon}^{\gamma}\left(\left|V_{T_{k-1}^{\epsilon, \zeta}}^{\epsilon, \zeta}-v_{T_{k}^{\epsilon, \zeta}}\left(\bar{R}_{k}^{\epsilon, \zeta}\right)\right|\right)\right\}} .
$$

For $t \in[0, T], w \in \mathbb{R}^{2}$, (recall that $\phi_{\epsilon} \leq \Gamma_{\epsilon}$ ), define

$$
\begin{aligned}
g_{\epsilon, \zeta}(t, w) & =1-\frac{1}{\lambda_{\epsilon, \zeta}} \int_{0}^{1} d \rho \int_{-G(\zeta)-1}^{G(\zeta)+1} d z \phi_{\epsilon}^{\gamma}\left(\left|w-v_{t}(\rho)\right|\right) \\
& =1-\frac{1}{2 \Gamma_{\epsilon}^{\gamma}} \int_{0}^{1} d \rho \phi_{\epsilon}^{\gamma}\left(\left|w-v_{t}(\rho)\right|\right) \in[1 / 2,1] .
\end{aligned}
$$

Consider a $C^{\infty}$ function $\chi: \mathbb{R} \mapsto[0,1]$ supported by $(-1,1)$ such that $\int_{-1}^{1} \chi(x) d x=1$. Setting

$$
q_{\epsilon, \zeta}(t, w, \rho, z)=g_{\epsilon, \zeta}(t, w) \chi(z-G(\zeta)-3)+\frac{\phi_{\epsilon}^{\gamma}\left(\left|w-v_{t}(\rho)\right|\right)}{\lambda_{\epsilon, \zeta}} \mathbb{1}_{\{|z| \leq G(\zeta)+1\}}
$$

we see that for each $t \in[0, T], w \in \mathbb{R}^{2}, q_{\epsilon, \zeta}(t, w, \rho, z) d \rho d z$ is a probability measure on $[0,1] \times \mathbb{R}_{*}$. Since $\chi(z-G(\zeta)-3)=0$ for $|z| \leq G(\zeta)+1$ and $\chi(z-G(\zeta)-3)>0$ implies $|z|>G(\zeta)+1$ and thus $\mathbf{I}_{\zeta}(z)=0$, we see that for all $k \geq 0$, all $\psi: \mathbb{R}^{2} \mapsto \mathbb{R}_{+}$,

$$
\begin{aligned}
& \mathbb{E}\left[\psi\left(V_{T_{k+1}^{\epsilon, \zeta}}^{\epsilon, \zeta}\right) \mid V_{T_{k}^{\epsilon, \zeta}}^{\epsilon, \zeta}, T_{k}^{\epsilon, \zeta}, T_{k+1}^{\epsilon, \zeta}\right] \\
= & \int_{0}^{1} \int_{\mathbb{R}_{*}} \psi\left(V_{T_{k}^{\epsilon, \zeta}}^{\epsilon, \zeta}+A(\vartheta(z))\left(V_{T_{k}, \zeta}^{\epsilon, \zeta}-v_{T_{k+1}^{\epsilon, \zeta}}(\rho)\right) \mathbf{I}_{\zeta}(z)\right) \phi_{\epsilon}^{\gamma}\left(\left|V_{T_{k}^{\epsilon, \zeta}}^{\epsilon, \zeta}-v_{T_{k+1}^{\epsilon, \zeta}}(\rho)\right|\right) \frac{d \rho d z}{\lambda_{\epsilon, \zeta}} \\
= & \int_{0}^{1} \int_{\mathbb{R}_{*}} \psi\left(V_{T_{k}^{\epsilon, \zeta}}^{\epsilon, \zeta}+A(\vartheta(z))\left(V_{T_{k}}^{\epsilon, \zeta}-v_{T_{k+1}^{\epsilon, \zeta}}(\rho)\right) \mathbf{I}_{\zeta}(z)\right) q_{\epsilon, \zeta}\left(T_{k+1}^{\epsilon, \zeta}, V_{T_{k}^{\epsilon, \zeta}}^{\epsilon, \zeta}, \rho, z\right) d \rho d z .
\end{aligned}
$$

Consequently, we can build, on a possibly enlarged probability space, a sequence $\left(R_{k}^{\epsilon, \zeta}, Z_{k}^{\epsilon, \zeta}\right)_{k \geq 1}$ of random variables such that $V_{0}^{\epsilon, \zeta}=V_{0}$ and for all $k \in\left\{0, \ldots, J_{T}^{\epsilon, \zeta}-1\right\}$,

$$
\begin{aligned}
& V_{t}^{\epsilon, \zeta}=V_{T_{k}^{\epsilon, \zeta}}^{\epsilon, \zeta} \quad \text { for all } t \in\left[T_{k}^{\epsilon, \zeta}, T_{k+1}^{\epsilon, \zeta}\right), \\
& V_{T_{k+1}^{\epsilon, \zeta}}^{\epsilon, \zeta}=\sum_{k=1}^{J_{t}^{\epsilon, \zeta}} A\left(\vartheta\left(Z_{k+1}^{\epsilon, \zeta}\right)\right)\left(V_{T_{k}}^{\epsilon, \zeta}-v_{T_{k+1}^{\epsilon, \zeta}}\left(R_{k+1}^{\epsilon, \zeta}\right)\right) \mathbf{I}_{\zeta}\left(Z_{k+1}^{\epsilon, \zeta}\right), \\
& \mathcal{L}\left(\left(R_{k+1}^{\epsilon, \zeta}, Z_{k+1}^{\epsilon, \zeta}\right) \mid V_{T_{k}^{\epsilon, \zeta}}^{\epsilon, \zeta}, T_{k}^{\epsilon, \zeta}, T_{k+1}^{\epsilon, \zeta}\right)=q_{\epsilon, \zeta}\left(T_{k+1}^{\epsilon, \zeta}, V_{T_{k}^{\epsilon, \zeta}}^{\epsilon, \zeta}, \rho, z\right) d \rho d z .
\end{aligned}
$$

Observe that by construction, we have

$$
V_{t}^{\epsilon, \zeta}=V_{0}+\sum_{k=1}^{J_{t}^{\epsilon, \zeta}} A\left(\vartheta\left(Z_{k}^{\epsilon, \zeta}\right)\right)\left(V_{T_{k-1}^{\epsilon, \zeta}}^{\epsilon, \zeta}-v_{T_{k}^{\epsilon, \zeta}}\left(R_{k}^{\epsilon, \zeta}\right)\right) \mathbf{I}_{\zeta}\left(Z_{k}^{\epsilon, \zeta}\right)
$$

for all $t \in[0, T]$. The following observation will allow us to handle several computations. 
Remark 3.1. Recall that $M(d s, d \rho, d z, d u)$ is a Poisson measure on $[0, T] \times[0,1] \times \mathbb{R}_{*} \times[0, \infty)$ with intensity measure dsd $\rho d z d u$. For any $\psi:[0, T] \times \mathbb{R}^{2} \times[0,1] \times \mathbb{R}_{*} \mapsto \mathbb{R}_{+}$, any $t \in[0, T]$,

$$
\begin{aligned}
& \sum_{k=1}^{J_{t}^{\epsilon, \zeta}} \psi\left(T_{k}^{\epsilon, \zeta}, V_{T_{k-1}^{\epsilon, \zeta}}^{\epsilon, \zeta}, R_{k}^{\epsilon, \zeta}, Z_{k}^{\epsilon, \zeta}\right) \mathbf{I}_{\zeta}\left(Z_{k}^{\epsilon, \zeta}\right) \\
= & \int_{0}^{t} \int_{0}^{1} \int_{\mathbb{R}_{*}} \int_{0}^{\infty} \psi\left(s, V_{s-}^{\epsilon, \zeta}, \rho, z\right) \mathbf{I}_{\zeta}(z) \mathbb{I}_{\left\{u \leq \phi_{\epsilon}^{\gamma}\left(\left|V_{s-}^{\epsilon, \zeta}-v_{s}(\rho)\right|\right)\right\}} M(d s, d \rho, d z, d u) .
\end{aligned}
$$

We conclude this section with the computation of the law of $\left(\left(R_{1}^{\epsilon, \zeta}, Z_{1}^{\epsilon, \zeta}\right), \ldots,\left(R_{l}^{\epsilon, \zeta}, Z_{l}^{\epsilon, \zeta}\right)\right)$.

Remark 3.2. We can write, for each $k \geq 0$,

$$
V_{T_{k}}^{\epsilon, \zeta}=\mathcal{H}_{k}\left(V_{0},\left(T_{1}^{\epsilon, \zeta}, R_{1}^{\epsilon, \zeta}, Z_{1}^{\epsilon, \zeta}\right), \ldots,\left(T_{k}^{\epsilon, \zeta}, R_{k}^{\epsilon, \zeta}, Z_{k}^{\epsilon, \zeta}\right)\right),
$$

for some function $\mathcal{H}_{k}: \mathbb{R}^{2} \times\left(\mathbb{R}_{+} \times[0,1] \times \mathbb{R}_{*}\right)^{k} \mapsto \mathbb{R}^{2}$. Indeed, set $\mathcal{H}_{0}(v)=v$ and

$$
\begin{aligned}
\left.\mathcal{H}_{k+1}\left(v,\left(t_{1}, \rho_{1}, z_{1}\right), \ldots,\left(t_{k+1}, \rho_{k+1}, z_{k+1}\right)\right)=\mathcal{H}_{k}\left(v,\left(t_{1}, \rho_{1}, z_{1}\right), \ldots,\left(t_{k}, \rho_{k}, z_{k}\right)\right)\right) \\
\left.+A\left(\vartheta\left(z_{k+1}\right)\right)\left(\mathcal{H}_{k}\left(v,\left(t_{1}, \rho_{1}, z_{1}\right), \ldots,\left(t_{k}, \rho_{k}, z_{k}\right)\right)\right)-v_{t_{k+1}}\left(\rho_{k+1}\right)\right) \mathbf{I}_{\zeta}\left(z_{k+1}\right) .
\end{aligned}
$$

Conditionally on $\sigma\left(V_{0}, J_{t}^{\epsilon, \zeta}, t \geq 0\right)$, the law of $\left(\left(R_{1}^{\epsilon, \zeta}, Z_{1}^{\epsilon, \zeta}\right), \ldots,\left(R_{l}^{\epsilon, \zeta}, Z_{l}^{\epsilon, \zeta}\right)\right)$ has the density

$$
\prod_{k=1}^{l} q_{\epsilon, \zeta}\left(T_{k}^{\epsilon, \zeta}, \mathcal{H}_{k-1}\left(V_{0},\left(T_{1}^{\epsilon, \zeta}, \rho_{1}, z_{1}\right), \ldots,\left(T_{k-1}^{\epsilon, \zeta}, \rho_{k-1}, z_{k-1}\right)\right), \rho_{k}, z_{k}\right),
$$

with respect to the Lebesgue measure on $\left([0,1] \times \mathbb{R}_{*}\right)^{k}$.

\section{An integration by parts Formula}

The aim of this section is to prove the following integration by parts formula for $V_{t}^{\epsilon, \zeta}$. Clearly, on the event $\left\{T_{1}^{\epsilon, \zeta}>t\right\}, V_{t}^{\epsilon, \zeta}=V_{0}$, so that no regularization may occur. To avoid this degeneracy, we consider $\left(Z_{-1}, Z_{0}\right)$ with law $\mathcal{N}\left(0, I_{2}\right)$ independent of everything else. We also introduce a $C^{\infty}$ non-decreasing function $\Phi_{\epsilon}: \mathbb{R} \mapsto[0,1]$ such that $\Phi_{\epsilon}(x)=0$ for $x \leq \Gamma_{\epsilon}-1$ and $\Phi_{\epsilon}(x)=1$ for $x \geq \Gamma_{\epsilon}$. We may assume that the derivatives of all orders of $\Phi_{\epsilon}$ are bounded uniformly with respect to $\epsilon \in\left(0, \epsilon_{0}\right)$. Finally, we consider a $C^{\infty}$ function $\Psi: \mathbb{R} \mapsto[0,1]$ such that $\Psi(x)=1$ for $x \leq 1 / 4$ and $\Psi(x)=0$ for $x \geq 3 / 4$. We set

$$
\Sigma_{t}^{\epsilon, \zeta}=\Phi_{\epsilon}\left(\left|V_{0}\right|\right)+\sum_{k=1}^{J_{t}^{\epsilon, \zeta}} \Phi_{\epsilon}\left(\left|V_{T_{k}^{\epsilon, \zeta}}^{\epsilon, \zeta}\right|\right) \quad \text { and } \quad G_{t}^{\epsilon, \zeta}=\Psi\left(\Sigma_{t}^{\epsilon, \zeta}\right) .
$$

Observe that since $\sup _{[0, t]}\left|V_{s}^{\epsilon, \zeta}\right|=\max \left\{\left|V_{0}\right|,\left|V_{T_{1}^{\epsilon, \zeta}}^{\epsilon, \zeta}\right|, \ldots,\left|V_{T_{J_{t}}^{\epsilon, \zeta}}^{\epsilon, \zeta}\right|\right\}$, we have

$$
\mathbb{I}_{\left\{\sup _{[0, t]}\left|V_{s}^{\epsilon, \zeta}\right| \leq \Gamma_{\epsilon}-1\right\}} \leq G_{t}^{\epsilon, \zeta} \leq \mathbb{I}_{\left\{\sup _{[0, t]}\left|V_{s}^{\epsilon, \zeta}\right| \leq \Gamma_{\epsilon}\right\}} .
$$

Theorem 4.1. We set $u_{\zeta}(t):=t \zeta^{4+\nu}$. For any $\psi \in C_{b}^{\infty}\left(\mathbb{R}^{2}, \mathbb{R}\right)$, any $0<t_{0} \leq t \leq T$, any $\kappa \in\left(1 / \eta_{0}, \delta\right)$, any $q \geq 1$, any multi-index $\beta \in\{1,2\}^{q}$,

$$
\left|\mathbb{E}\left[\partial_{\beta}^{q} \psi\left(\sqrt{u_{\zeta}(t)}\left(\begin{array}{c}
Z_{-1} \\
Z_{0}
\end{array}\right)+V_{t}^{\epsilon, \zeta}\right) G_{t}^{\epsilon, \zeta}\right]\right| \leq C_{q, t_{0}, \kappa} e^{C_{q, \kappa} \Gamma_{\epsilon}^{\gamma}}\|\psi\|_{\infty}\left[\epsilon^{-q} \zeta^{-\nu q}+e^{-\Gamma_{\epsilon}^{\kappa} \zeta^{-2 \nu q}}\right] .
$$

In the whole section, $\zeta \in(0,1)$ and $\epsilon \in\left(0, \epsilon_{0}\right)$ are fixed. We set for simplicity $\lambda=\lambda_{\epsilon, \zeta}, T_{k}=T_{k}^{\epsilon, \zeta}$, $R_{k}=R_{k}^{\epsilon, \zeta}, Z_{k}=Z_{k}^{\epsilon, \zeta}$, but we track the dependance of all the constants with respect to $\epsilon$ and $\zeta$. 
4.1. The Malliavin calculus. We recall here the Malliavin calculus defined in 2]. This calculus is based on the variables $\left(Z_{k}\right)_{k \geq 1}$ (they correspond to the variables $\left(V_{k}\right)_{k \geq 1}$ in [2]). The $\sigma$-field with respect to which we will take conditional expectations is

$$
\mathcal{G}=\sigma\left(V_{0}, T_{k}, R_{k}, k \geq 1\right) .
$$

The calculus presented below is slightly different from the one used in [2]: there one employes as basic random variables $\left(R_{k}, Z_{k}\right)_{k \geq 1}$, while here we use only $\left(Z_{k}\right)_{k \geq 1}$. This is because we have no informations about the derivability of the coefficients of the equation with respect to $\rho$. We also notice that our coefficients depend on time, but since the bounds of the coefficients and of their derivatives are uniform with respect to time, the estimates from [2] hold in our framework.

Recall that $\left(Z_{-1}, Z_{0}\right)$ is independent of everything else and $\mathcal{N}\left(0, I_{2}\right)$-distributed. We set

$$
\mathbf{Z}_{t}=\left(Z_{-1}, Z_{0}, Z_{1}, \ldots, Z_{J_{t}}\right) .
$$

We now use Remark 3.2. Conditionally on $\mathcal{G}$, the law of $\mathbf{Z}_{t}$ has the following density with respect to the Lebesgue measure on $\mathbb{R}^{2} \times\left(\mathbb{R}_{*}\right)^{J_{t}}$ : setting $z=\left(z_{-1}, \ldots, z_{J_{t}}\right)$,

$$
p_{\epsilon, \zeta}(z)=\mathcal{W}_{t} e^{-\frac{\left|z_{-1}\right|^{2}+\left|z_{0}\right|^{2}}{2}} \prod_{k=1}^{J_{t}} q_{\epsilon, \zeta}\left(T_{k}, \mathcal{H}_{k-1}\left(V_{0},\left(T_{1}, R_{1}, z_{1}\right), \ldots,\left(T_{k-1}, R_{k-1}, z_{k-1}\right)\right), R_{k}, z_{k}\right),
$$

the normalization constant

$$
\mathcal{W}_{t}=\left(2 \pi \int_{[0,1]^{J_{t}}}\left[\prod_{k=1}^{J_{t}} q_{\epsilon, \zeta}\left(T_{k}, \mathcal{H}_{k-1}\left(V_{0},\left(T_{1}, R_{1}, z_{1}\right), \ldots,\left(T_{k-1}, R_{k-1}, z_{k-1}\right)\right), R_{k}, z_{k}\right)\right] d z_{1} \ldots d z_{J_{t}}\right)^{-1}
$$

being $\mathcal{G}$-measurable.

We denote by $U_{\zeta}: \mathbb{R}_{*} \mapsto[0,1]$ a $C^{\infty}$ function such that $U_{\zeta}(z)=1$ for $|z| \in(1, G(\zeta)-1)$ and $U_{\zeta}(z)=0$ for $|z| \leq 1 / 2$ and $|z| \geq G(\zeta)-1 / 2$. We may of course choose $U_{\zeta}$ in such a way that its derivatives of all orders are uniformly bounded (with respect to $\zeta$ ). Then we define

$$
\pi_{-1}=\pi_{0}=1, \quad \pi_{k}=U_{\zeta}\left(Z_{k}\right), \quad k \geq 1 .
$$

Remark 4.2. Notice that $\pi_{k}$ is smooth with respect to $Z_{k}$ and that all its derivatives are bounded uniformly with respect to $\zeta$. This is the reason why we used the substition $\theta=\vartheta(z)$ in the previous section.

A simple functional is a random variable $F$ of the form

$$
F=h\left(\omega,\left(Z_{-1}, \ldots, Z_{J_{t}}\right)\right)=h\left(\omega, \mathbf{Z}_{t}\right)
$$

for some $t \geq 0$, some $\mathcal{G}$-measurable $h:\left\{(\omega, z), \omega \in \Omega, z \in \mathbb{R}^{2} \times\left(\mathbb{R}_{*}\right)^{J_{t}(\omega)}\right\} \mapsto \mathbb{R}$, such that for almost all $\omega \in \Omega$, for all $k \in\left\{-1, \ldots, J_{t}(\omega)\right\}, z \mapsto f(\omega, z)$ is smooth with respect to $z_{k}$ on the set $\pi_{k}>0$. For such a functional we define the Malliavin derivatives: for $k \geq-1$,

$$
D_{k} F=\pi_{k} \partial_{z_{k}} h\left(\omega, \mathbf{Z}_{t}\right) .
$$

Remark 4.3. We notice that Remark 3.2 ensures us that $V_{t}^{\epsilon, \zeta}$ is a simple functionnal for each $t \in[0, T]$. Indeed, $\mathcal{H}_{k}$ is smooth with respect to $z_{l}$ for $l \in\{1, \ldots, k\}$ on $\left\{z_{l} \in(-G(\zeta), 0) \cup(0, G(\zeta))\right.$, which contains $\left\{\pi_{l}>0\right\}$. This explains our choice for $\pi_{l}$.

Observe that if $F$ is a simple functional, $D_{k} F$ is also a simple functional (in particular because the weights $\pi_{k}$ are smooth functions of $\left.Z\right)$. Thus for a multi-index $\beta=\left(k_{1}, \ldots, k_{m}\right)$ with length $|\beta|=m$, we may define

$$
D^{\beta} F=D_{k_{m}} \ldots D_{k_{1}} F .
$$


For $m \geq 1$, we will use the norm

$$
|F|_{m}=|F|+\sum_{1 \leq|\beta| \leq m}\left|D^{\beta} F\right| .
$$

Given a $d$-dimensional random variable $F=\left(F_{1}, \ldots, F_{d}\right)$ we set $|F|_{m}=\sum_{i=1}^{d}\left|F_{i}\right|_{m}$. The Malliavin covariance matrix of $F$ is defined by

$$
\sigma^{i, j}(F)=\sum_{k=-1}^{J_{t}} D_{k} F_{i} \times D_{k} F_{j}, \quad 1 \leq i, j \leq d .
$$

Finally, we introduce the divergence operator $L$ : for a simple functional $F$,

$$
L F=-\sum_{k=-1}^{J_{t}}\left[\frac{1}{\pi_{k}} D_{k}\left(\pi_{k} D_{k} F\right)+D_{k} F \times D_{k} \log p_{\varepsilon, \zeta}\left(\mathbf{Z}_{t}\right)\right] .
$$

We now are able to state the integration by parts formula obtained in [2, Theorems 1 and 3], of which the assumptions are satisfied. Let $G$ and $F=\left(F_{1}, \ldots, F_{d}\right)$ be simple functionals. We suppose that $\operatorname{det} \sigma(F) \neq 0$ almost surely. Then for every $\psi \in C_{b}^{\infty}\left(\mathbb{R}^{d}, \mathbb{R}\right)$ and every multi-index $\beta=\left(\beta_{1}, \ldots, \beta_{q}\right) \in\{1, \ldots, d\}^{q}$, we have

$$
\mathbb{E}\left(\partial_{\beta}^{q} \psi(F) G\right)=\mathbb{E}\left(\psi(F) K_{\beta, q}(F, G)\right),
$$

with the following estimate:

$$
\left|K_{\beta, q}(F, G)\right| \leq C_{q, d} \frac{|G|_{q}\left(1+|F|_{q+1}\right)^{q(6 d+1)}}{|\operatorname{det} \sigma(F)|^{3 q-1}}\left(1+\sum_{j=1}^{q} \sum_{k_{1}+. .+k_{j} \leq q-j} \prod_{i=1}^{j}|L F|_{k_{i}}\right) .
$$

4.2. Lower-bound of the covariance matrix. The aim of this subsection is to show the following proposition. We denote by $I$ the identity matrix of $M_{2 \times 2}(\mathbb{R})$. As we will see below (see Subsection 4.4), the Malliavin covariance matrix of $\sqrt{u_{\zeta}(t)}\left(\begin{array}{c}Z_{-1} \\ Z_{0}\end{array}\right)+V_{t}^{\epsilon, \zeta}$ is nothing but $u_{\zeta}(t) I+\sigma\left(V_{t}^{\epsilon, \zeta}\right)$.

Proposition 4.4. Recall that $u_{\zeta}(t):=t \zeta^{4+\nu}$. For all $p \geq 1$, all $0<t_{0}<t<T$,

$$
\mathbb{E}\left[\left(\operatorname{det}\left[u_{\zeta}(t) I+\sigma\left(V_{t}^{\epsilon, \zeta}\right)\right]\right)^{-p}\right] \leq C_{t_{0}, p} e^{C_{p} \Gamma_{\epsilon}^{\gamma}} .
$$

First, we compute the derivatives of $V_{t}^{\epsilon, \zeta}$ for $t \in[0, T]$. If we have a family $\left(M_{k}\right)_{k \in\{1, \ldots, j\}}$ in $M_{2 \times 2}(\mathbb{R})$, we write $\prod_{k=1}^{j} M_{k}=M_{j} \ldots M_{1}$.

Lemma 4.5. Let $\left(Y_{t}\right)_{t \in[0, T]}$ be the $M_{2 \times 2}(\mathbb{R})$-valued process defined by

$$
Y_{t}=\prod_{k=1}^{J_{t}}\left[I+A\left(\vartheta\left(Z_{k}\right)\right) \mathbf{I}_{\zeta}\left(Z_{k}\right)\right] \quad\left(\text { with } Y_{t}=I \text { if } J_{t}=0\right) .
$$

This process solves

$$
Y_{t}=I+\sum_{k=1}^{J_{t}} A\left(\vartheta\left(Z_{k}\right)\right) \mathbf{I}_{\zeta}\left(Z_{k}\right) Y_{T_{k-1}}
$$

and $Y_{t}$ is invertible for all $t \in[0, T]$, because $I+A(\theta)$ is invertible for $|\theta| \leq \pi / 2$. Set, for $k \geq 1$,

$$
H_{k}=\vartheta^{\prime}\left(Z_{k}\right) A^{\prime}\left(\vartheta\left(Z_{k}\right)\right)\left(V_{T_{k-1}}^{\epsilon, \zeta}-v_{T_{k}}\left(R_{k}\right)\right) .
$$


Then for $k \geq 1$, for $t \in[0, T]$,

$$
D_{k} V_{t}^{\epsilon, \zeta}=\pi_{k} Y_{t} Y_{T_{k}}^{-1} H_{k} \mathbb{I}_{t \geq T_{k}} .
$$

Proof. Since $V_{t}^{\epsilon, \zeta}$ and $Y_{t}$ are constant on $\left[T_{j}, T_{j+1}\right)$, it suffices to check the result for $V_{T_{j}}^{\epsilon, \zeta}$, for all $j \geq 0$, that is, on the set $\pi_{k}>0$ (i.e. $\left|Z_{k}\right| \in[1 / 2, G(\zeta)-1 / 2]$ ),

$$
\partial_{z_{k}} V_{T_{j}}^{\epsilon, \zeta}=Y_{T_{j}} Y_{T_{k}}^{-1} H_{k} \mathbb{I}_{j \geq k} .
$$

Since $V_{T_{j}}^{\epsilon, \zeta}$ does not depend on $Z_{k}$ if $j<k$, the result is obvious for $j<k$. We now work by induction on $j \geq k$. First, $V_{T_{k}}^{\epsilon, \zeta}=V_{T_{k-1}}^{\epsilon, \zeta}+A\left(\vartheta\left(Z_{k}\right)\right)\left(V_{T_{k-1}}^{\epsilon, \zeta}-v_{T_{k}}\left(R_{k},\right)\right) \mathbf{I}_{\zeta}\left(Z_{k}\right)$. Derivating this formula with respect to $z_{k}$ yields (recall that $\left|Z_{k}\right| \in[1 / 2, G(\zeta-1 / 2)]$ and thus $\mathbf{I}_{\zeta}\left(Z_{k}\right)=1$ ),

$$
\partial_{z_{k}} V_{T_{k}}^{\epsilon, \zeta}=\vartheta^{\prime}\left(Z_{k}\right) A^{\prime}\left(\vartheta\left(Z_{k}\right)\right)\left(V_{T_{k-1}}^{\epsilon, \zeta}-v_{T_{k}}\left(R_{k}\right)\right)=Y_{T_{k}} Y_{T_{k}}^{-1} H_{k} .
$$

We now assume that the result holds for some $j \geq k$ and we recall that due to Section 3 , $V_{T_{j+1}}^{\epsilon, \zeta}=$ $V_{T_{j}}^{\epsilon, \zeta}+A\left(\vartheta\left(Z_{j+1}\right)\right)\left(V_{T_{j}}^{\epsilon, \zeta}-v_{T_{j+1}}\left(R_{j+1}\right)\right) \mathbf{I}_{\zeta}\left(Z_{j+1}\right)$. Hence

$$
\begin{aligned}
\partial_{z_{k}} V_{T_{j+1}}^{\epsilon, \zeta} & =\left(I+A\left(\vartheta\left(Z_{j+1}\right)\right) \mathbf{I}_{\zeta}\left(Z_{j+1}\right)\right) \partial_{z_{k}} V_{T_{j}}^{\epsilon, \zeta} \\
& =\left(I+A\left(\vartheta\left(Z_{j+1}\right)\right) \mathbf{I}_{\zeta}\left(Z_{j+1}\right)\right) Y_{T_{j}} Y_{T_{k}}^{-1} H_{k}=Y_{T_{j+1}} Y_{T_{k}}^{-1} H_{k}
\end{aligned}
$$

as desired.

We deduce the following expression.

Lemma 4.6. For all $t \in[0, T], \sigma\left(V_{t}^{\epsilon, \zeta}\right)=Y_{t} S_{t} Y_{t}^{*}$, where

$$
S_{t}:=\sum_{k=1}^{J_{t}} \pi_{k}^{2} Y_{T_{k}}^{-1} H_{k} H_{k}^{*}\left(Y_{T_{k}}^{-1}\right)^{*}
$$

Proof. Due to Lemma 4.5, we have

$$
\sigma\left(V_{t}^{\epsilon, \zeta}\right)=\sum_{k=1}^{J_{t}} \pi_{k}^{2}\left[Y_{t} Y_{T_{k}}^{-1} H_{k}\right]\left[Y_{t} Y_{T_{k}}^{-1} H_{k}\right]^{*}=Y_{t}\left(\sum_{k=1}^{J_{t}} \pi_{k}^{2} Y_{T_{k}}^{-1} H_{k} H_{k}^{*}\left(Y_{T_{k}}^{-1}\right)^{*}\right) Y_{t}^{*},
$$

whence the result.

Next, we prove some estimates concerning $\left(Y_{t}\right)_{t \in[0, T]}$.

Lemma 4.7. Almost surely, for all $t \geq 0,\left|Y_{t}\right| \leq 1$. Furthermore, for all $p \geq 1$,

$$
\mathbb{E}\left[\sup _{[0, T]}\left|Y_{t}^{-1}\right|^{p}\right] \leq \exp \left(C_{p} \Gamma_{\epsilon}^{\gamma}\right)
$$

Proof. First, an immediate computation shows that

$$
|I+A(\theta)|^{2}=\sup _{|\xi|=1}|(I+A(\theta)) \xi|^{2}=\frac{1+\cos \theta}{2} \leq 1,
$$

so that $\left|Y_{t}\right| \leq 1$. Next, one can check that for $\theta \in(-\pi / 2, \pi / 2)$,

$$
\left|(I+A(\theta))^{-1}\right|^{2}=\frac{2}{1+\cos \theta} \leq 1+\theta^{2} \leq \exp \left(\theta^{2}\right) .
$$


Thus for $0 \leq t \leq T$,

$$
\left|Y_{t}^{-1}\right|^{2} \leq \prod_{k=1}^{J_{t}}\left|\left(I+A\left(\vartheta\left(Z_{k}\right)\right) \mathbf{I}_{\zeta}\left(Z_{k}\right)\right)^{-1}\right|^{2} \leq \exp \left(\sum_{k=1}^{J_{T}} \vartheta^{2}\left(Z_{k}\right) \mathbf{I}_{\zeta}\left(Z_{k}\right)\right)=: \exp \left(L_{T}\right) .
$$

We infer from Remark 3.1 that for some Poisson measure $M$ with intensity measure $d s d \rho d z d u$,

$$
\begin{aligned}
L_{T} & =\int_{0}^{T} \int_{0}^{1} \int_{\mathbb{R}_{*}} \int_{0}^{\infty}|\vartheta(z)|^{2} \mathbf{I}_{\zeta}(z) \mathbb{I}_{\left\{u \leq \phi_{\epsilon}^{\gamma}\left(\left|V_{s-}^{\epsilon, \zeta}-v_{s}(\rho)\right|\right)\right\}} M(d s, d \rho, d z, d u) \\
& \leq \int_{0}^{T} \int_{0}^{1} \int_{\mathbb{R}_{*}} \int_{0}^{\infty}|\vartheta(z)|^{2} \mathbb{I}_{\left\{u \leq \Gamma_{\epsilon}^{\gamma}\right\}} M(d s, d \rho, d z, d u) .
\end{aligned}
$$

Hence for any $p>0$,

$$
\mathbb{E}\left[\exp \left(p L_{T}\right)\right] \leq \exp \left(\Gamma_{\epsilon}^{\gamma} T \int_{\mathbb{R}_{*}}\left(e^{p \vartheta^{2}(z)}-1\right) d z\right) \leq \exp \left(C_{p} T \Gamma_{\epsilon}^{\gamma}\right)
$$

since $\vartheta^{2}(z) \leq(\pi / 2)^{2}$ and since $\int_{\mathbb{R}_{*}} \vartheta^{2}(z) d z=\int_{-\pi / 2}^{\pi / 2} \theta^{2} b(\theta) d \theta<\infty$ by (3.2) and $(\mathbf{A}(\gamma, \nu))$.

To bound $S_{t}$ from below, we need a lower-bound of $f_{t}$. Recall (3.1).

Lemma 4.8. One may find $r_{0}>0$ and $q_{0}>0$ such that for any $w \in \mathbb{R}^{2}$, any $t \in[0, T]$,

$$
f_{t}\left(\left\{v,|v-w| \geq r_{0}\right\}\right)=\int_{0}^{1} \mathbb{1}_{\left\{\left|v_{t}(\rho)-w\right| \geq r_{0}\right\}} d \rho \geq q_{0} .
$$

Proof. Recall that by (1.8), we have $\int_{\mathbb{R}^{2}}|v|^{2} f_{t}(d v)=e_{0}>0$ and $\int_{\mathbb{R}^{2}} v f_{t}(d v)=0$. First, we observe that for all $w$ such that $|w| \geq \sqrt{2 e_{0}}+1=: a$, we have

$f_{t}(\{v,|v-w| \geq 1\}) \geq f_{t}(\{v,|v| \leq|w|-1\})=1-f_{t}(\{v,|v|>|w|-1\}) \geq 1-e_{0} /(|w|-1)^{2} \geq 1 / 2$.

Thus it suffices to prove the result for $(t, w) \in[0, T] \times \overline{\operatorname{Ball}(0, a)}$. We notice that for each $t \geq 0$, $f_{t}$ is not a Dirac mass. Indeed, since $\int_{\mathbb{R}^{2}} v f_{t}(d v)=0$, the only possible Dirac mass is $\delta_{0}$, but this would imply $\int_{\mathbb{R}^{2}}|v|^{2} f_{t}(d v)=0$.

As a consequence, we can find, for each $(t, w) \in[0, T] \times \overline{\operatorname{Ball}(0, a)}$, some numbers $r_{t, w}>0$ and $q_{t, w}>0$ such that $f_{t}\left(\left\{v,|v-w| \geq r_{t, w}\right\}\right) \geq q_{t, w}$.

Now we prove that for each $(t, w) \in[0, T] \times \overline{\operatorname{Ball}(0, a)}$, we can find a neighborhood $\mathcal{V}_{t, w}$ of $(t, w)$ such that for all $\left(t^{\prime}, w^{\prime}\right) \in \mathcal{V}_{t, w}, f_{t^{\prime}}\left(\left\{v,\left|v-w^{\prime}\right| \geq r_{t, w} / 2\right\}\right) \geq q_{t, w} / 2$. To do so, we first observe that it is clear from Definition 1.1 that $t \mapsto f_{t}$ is weakly continuous. Hence for all continuousbounded function $\varphi: \mathbb{R} \mapsto \mathbb{R}_{+},\left(t^{\prime}, w^{\prime}\right) \mapsto \int_{\mathbb{R}^{2}} \varphi\left(\left|w^{\prime}-v\right|\right) f_{t^{\prime}}(d v)$ is continuous. Consider now a continuous-bounded nonnegative function $\varphi: \mathbb{R}_{+} \mapsto \mathbb{R}_{+}$such that $\mathbb{I}_{\left\{x \geq r_{t, w}\right\}} \leq \varphi \leq \mathbb{I}_{\left\{x \geq r_{t, w} / 2\right\}}$. By continuity, there is a neighborhood $\mathcal{V}_{t, w}$ of $(t, w)$ such that for all $\left(t^{\prime}, w^{\prime}\right) \in \mathcal{V}_{t, w}$, there holds $\int_{\mathbb{R}^{2}} \varphi\left(\left|w^{\prime}-v\right|\right) f_{t^{\prime}}(d v) \geq \frac{1}{2} \int_{\mathbb{R}^{2}} \varphi(|w-v|) f_{t}(d v)$, which implies

$$
f_{t^{\prime}}\left(\left\{v,\left|v-w^{\prime}\right| \geq r_{t, w} / 2\right\}\right) \geq \frac{1}{2} f_{t}\left(\left\{v,|v-w| \geq r_{t, w}\right\}\right) \geq q_{t, w} / 2 .
$$

Since $[0, T] \times \overline{\operatorname{Ball}(0, a)}$ is compact, we can find a finite covering $[0, T] \times \overline{\operatorname{Ball}(0, a)} \subset \cup_{i=1}^{n} \mathcal{V}_{t_{i}, w_{i}}$. We conclude choosing $r_{0}=\min \left(r_{t_{i}, w_{i}} / 2\right) \wedge 1$ and $q_{0}=\min \left(q_{t_{i}, w_{i}} / 2\right) \wedge(1 / 2)$.

We carry on with some basic but fundamental considerations. 
Lemma 4.9. For $\xi \in \mathbb{R}^{2}, X \in \mathbb{R}^{2}$, consider

$$
I(\xi, X)=\left\{\theta \in[-\pi / 2, \pi / 2],\left\langle\xi,(I+A(\theta))^{-1} A^{\prime}(\theta) X\right\rangle^{2} \geq \theta^{2}|X|^{2}|\xi|^{2} / 128\right\} .
$$

For any $\xi, X \in \mathbb{R}^{2}$, we always have either $(0, \pi / 2] \subset I(\xi, X)$ or $[-\pi / 2,0) \subset I(\xi, X)$.

Proof. We may assume, by homogeneity, that $|X|=|\xi|=1$. We have

$$
\begin{aligned}
& (I+A(\theta))^{-1} A^{\prime}(\theta)=\frac{1}{2}\left(\begin{array}{cc}
\frac{-\sin \theta}{1+\cos \theta} & -1 \\
1 & \frac{-\sin \theta}{1+\cos \theta}
\end{array}\right)=: \frac{1}{2}\left[\frac{-\sin \theta}{1+\cos \theta} I+P\right], \\
& \left\langle\xi,(I+A(\theta))^{-1} A^{\prime}(\theta) X\right\rangle^{2}=\frac{1}{4}\left[\frac{\sin ^{2} \theta}{(1+\cos \theta)^{2}}\langle\xi, X\rangle^{2}+\langle\xi, P X\rangle^{2}-2 \frac{\sin \theta}{1+\cos \theta}\langle\xi, X\rangle\langle\xi, P X\rangle\right] .
\end{aligned}
$$

Since $\langle X, P X\rangle=0$ and $|X|=|\xi|=1$, we always have either $\langle\xi, X\rangle^{2} \geq 1 / 2$ or $\langle\xi, P X\rangle^{2} \geq 1 / 2$. Thus for all $\theta$ such that $\langle\xi, X\rangle\langle\xi, P X\rangle \sin \theta \leq 0$ (this holds either on $[0, \pi / 2]$ or on $[-\pi / 2,0]$ ),

$$
\left\langle\xi,(I+A(\theta))^{-1} A^{\prime}(\theta) X\right\rangle^{2} \geq \frac{1}{8} \min \left[\frac{\sin ^{2} \theta}{(1+\cos \theta)^{2}}, 1\right] \geq \frac{\sin ^{2} \theta}{32} .
$$

We easily conclude, since $|\sin \theta| \geq|\theta| / 2$ on $[-\pi / 2, \pi / 2]$.

We deduce the following estimate.

Lemma 4.10. There are some constants $c>0, C>0$ such that for all $\xi \in \mathbb{R}^{2}$, all $t \in[0, T]$,

$$
\mathbb{E}\left[\exp \left(-\xi^{*} S_{t} \xi\right)\right] \leq C \exp \left(-c t\left[|\xi|^{\nu /(2+\nu)} \wedge \zeta^{-\nu}\right]\right) .
$$

Proof. Recalling Lemmas 4.5, 4.6, the definition of $\pi_{k}$ and using that $Y_{T_{k}}=\left(I+A\left(\vartheta\left(Z_{k}\right)\right)\right) Y_{T_{k-1}}$ on $\pi_{k}>0$ (because $\pi_{k}>0$ implies $\mathbf{I}_{\zeta}\left(Z_{k}\right)=1$ ), we see that

$$
\begin{aligned}
& \xi^{*} S_{t} \xi=\sum_{k=1}^{J_{t}} \pi_{k}^{2}\left\langle Y_{T_{k}}^{-1} H_{k}, \xi\right\rangle^{2}=\sum_{k=1}^{J_{t}} \pi_{k}^{2}\left\langle\left(I+A\left(\vartheta\left(Z_{k}\right)\right)\right)^{-1} H_{k},\left(Y_{T_{k-1}}^{-1}\right)^{*} \xi\right\rangle^{2} \\
& \geq \sum_{k=1}^{J_{t}} \mathbb{I}_{\left\{\left|Z_{k}\right| \in[1 / 2, G(\zeta)-1 / 2]\right\}}\left(\vartheta^{\prime}\left(Z_{k}\right)\right)^{2}\left\langle\left(I+A\left(\vartheta\left(Z_{k}\right)\right)\right)^{-1} A^{\prime}\left(\vartheta\left(Z_{k}\right)\right)\left(V_{T_{k-1}, \zeta}^{\epsilon}-v_{T_{k}}\left(R_{k}\right)\right), \xi_{T_{k-1}}\right\rangle^{2},
\end{aligned}
$$

where $\xi_{t}:=\left(Y_{t}^{-1}\right)^{*} \xi$. We observe that a.s., $\left|\xi_{t}\right| \geq|\xi|$ because $\left|Y_{t}\right| \leq 1$ by Lemma 4.7 We splitted $Y_{T_{k}}=\left(I+A\left(\vartheta\left(Z_{k}\right)\right)\right) Y_{T_{k-1}}$ in order to make rigorous the stochastic calculus below $\left(\xi_{T_{k-1}}\right.$ will be predictable). We recall that $r_{0}$ and $q_{0}$ were defined in Lemma 4.8. Thus, due to Lemma 4.9.

$$
\begin{aligned}
& \xi^{*} S_{t} \xi \geq \sum_{k=1}^{J_{t}} \mathbb{I}_{\left\{\left|Z_{k}\right| \in[1 / 2, G(\zeta)-1 / 2]\right\}} \mathbb{I}_{\left\{\vartheta\left(Z_{k}\right) \in I\left(\xi_{T_{k-1}}, V_{T_{k-1}}^{\epsilon, \zeta}-v_{T_{k}}\left(R_{k}\right)\right)\right\}} \mathbb{I}_{\left\{\left|V_{T_{k-1}}^{\epsilon, \zeta}-v_{T_{k}}\left(R_{k}\right)\right| \geq r_{0}\right\}} \times \frac{\left(\vartheta^{\prime}\left(Z_{k}\right)\right)^{2} \vartheta^{2}\left(Z_{k}\right) r_{0}^{2}\left|\xi_{T_{k-1}}\right|^{2}}{128} \\
& \geq \frac{|\xi|^{2} r_{0}^{2}}{128} \sum_{k=1}^{J_{t}} \mathbb{1}_{\left\{\left|Z_{k}\right| \in[1 / 2, G(\zeta)-1 / 2]\right\}} \mathbb{I}_{\left\{\vartheta\left(Z_{k}\right) \in I\left(\xi_{T_{k-1}}, V_{T_{k-1}}^{\epsilon, \zeta}-v_{T_{k}}\left(R_{k}\right)\right)\right\}} \\
& \times \mathbb{1}_{\left.\left\{\mid V_{T_{k-1}}^{\epsilon, \zeta}-v_{T_{k}}\left(R_{k}\right)\right) \mid \geq r_{0}\right\}}\left(\vartheta^{\prime}\left(Z_{k}\right)\right)^{2} \vartheta^{2}\left(Z_{k}\right) \\
&=\frac{|\xi|^{2} r_{0}^{2}}{128} \int_{0}^{t} \int_{0}^{1} \int_{\mathbb{R}_{*}} \int_{0}^{\infty} \vartheta^{2}(z)\left(\vartheta^{\prime}(z)\right)^{2} \mathbb{I}_{\{|z| \in[1 / 2, G(\zeta)-1 / 2]\}} \mathbb{I}_{\left\{\vartheta(z) \in I\left(\xi_{s-}, V_{s-}^{\epsilon, \zeta}-v_{s}(\rho)\right)\right\}} \\
& \mathbb{1}_{\left\{\left|V_{s-}^{\epsilon, \zeta}-v_{s}(\rho)\right| \geq r_{0}\right\}} \mathbb{I}_{\left\{u \leq \phi_{\epsilon}^{\gamma}\left(\left|V_{s-}^{\epsilon, \zeta}-v_{s}(\rho)\right|\right)\right\}} M(d s, d \rho, d z, d u),
\end{aligned}
$$


where $M$ is a Poisson measure on $[0, T] \times[0,1] \times \mathbb{R}_{*} \times[0, \infty)$ with intensity measure $d s d \rho d z d u$. We used Remark 3.1. Since $\phi_{\epsilon}^{\gamma}(x) \geq r_{0}^{\gamma}$ for $x>r_{0}$ we get $\xi^{*} S_{t} \xi \geq \frac{|\xi|^{2} r_{0}^{2}}{128} L_{t}$, where

$$
\begin{array}{r}
L_{t}:=\int_{0}^{t} \int_{0}^{1} \int_{\mathbb{R}_{*}} \int_{0}^{\infty} \vartheta^{2}(z)\left(\vartheta^{\prime}(z)\right)^{2} \mathbb{I}_{\{|z| \in[1 / 2, G(\zeta)-1 / 2]\}} \mathbb{I}_{\left\{\vartheta(z) \in I\left(\xi_{s-}, V_{s-}^{\epsilon, \zeta}-v_{s}(\rho)\right)\right\}} \\
\mathbb{I}_{\left\{\left|V_{s-}^{\epsilon, \zeta}-v_{s}(\rho)\right| \geq r_{0}\right\}} \mathbb{I}_{\left\{u \leq r_{0}^{\gamma}\right\}} M(d s, d \rho, d z, d u) .
\end{array}
$$

Using the Itô formula for jump processes, taking expectations and differentiating with respect to time, we get, for $x>0$,

$$
\begin{aligned}
\frac{d}{d t} \mathbb{E}\left[e^{-x L_{t}}\right]=-\int_{0}^{1} \int_{\mathbb{R}_{*}} \int_{0}^{\infty} \mathbb{E}\left[e^{-x L_{t}}\left(1-e^{-x \vartheta^{2}(z)\left(\vartheta^{\prime}(z)\right)^{2}}\right) \mathbb{I}_{\{|z| \in[1 / 2, G(\zeta)-1 / 2]\}}\right. \\
\left.\mathbb{I}_{\left\{\vartheta(z) \in I\left(\xi_{t}, V_{t}^{\epsilon, \zeta}-v_{t}(\rho)\right)\right\}} \mathbb{I}_{\left\{\left|V_{t}^{\epsilon, \zeta}-v_{t}(\rho)\right| \geq r_{0}\right\}} \mathbb{I}_{\left\{u \leq r_{0}^{\gamma}\right\}}\right] d u d z d \rho .
\end{aligned}
$$

The integration with respect to $u$ is explicit. Using Lemma 4.9, we see that the set $\{\vartheta(z) \in$ $\left.I\left(\xi_{t}, V_{t}^{\epsilon, \zeta}-v_{t}(\rho)\right)\right\}$ a.s. contains $\{\vartheta(z) \in(0, \pi / 2)\}=\{z \in(0, \infty)\}$ or $\{\vartheta(z) \in(-\pi / 2,0)\}=\{z \in$ $(-\infty, 0)\}$. Since $\left(\vartheta \vartheta^{\prime}\right)^{2}$ is even, this yields

$$
\frac{d}{d t} \mathbb{E}\left[e^{-x L_{t}}\right] \leq-r_{0}^{\gamma} \int_{0}^{1} \int_{1 / 2}^{G(\zeta)-1 / 2} \mathbb{E}\left[e^{-x L_{t}}\left(1-e^{-x \vartheta^{2}(z)\left(\vartheta^{\prime}(z)\right)^{2}}\right) \mathbb{I}_{\left\{\left|V_{t}^{\epsilon, \zeta}-v_{t}(\rho)\right| \geq r_{0}\right\}}\right] d z d \rho
$$

Finally we use Lemma 4.8 to deduce

$$
\frac{d}{d t} \mathbb{E}\left[e^{-x L_{t}}\right] \leq-\left(r_{0}^{\gamma} q_{0} \int_{1 / 2}^{G(\zeta)-1 / 2}\left(1-e^{-x \vartheta^{2}(z)\left(\vartheta^{\prime}(z)\right)^{2}}\right) d z\right) \mathbb{E}\left[e^{-x L_{t}}\right] .
$$

Since $L_{0}=0$, this implies

$$
\mathbb{E}\left[e^{-x L_{t}}\right] \leq \exp \left(-t r_{0}^{\gamma} q_{0} \int_{1 / 2}^{G(\zeta)-1 / 2}\left(1-e^{-x \vartheta^{2}(z)\left(\vartheta^{\prime}(z)\right)^{2}}\right) d z\right) .
$$

Recalling that $\xi^{*} S_{t} \xi \geq \frac{|\xi|^{2} r_{0}^{2}}{128} L_{t}$, we get

$$
\mathbb{E}\left[\exp \left(-\xi^{*} S_{t} \xi\right)\right] \leq \exp \left(-t r_{0}^{\gamma} q_{0} \int_{1 / 2}^{G(\zeta)-1 / 2}\left(1-e^{-|\xi|^{2} r_{0}^{2} \vartheta^{2}(z)\left(\vartheta^{\prime}(z)\right)^{2} / 128}\right) d z\right) .
$$

We observe that due to $(\mathbf{A}(\gamma, \nu))$,

$$
G(\zeta)-1 / 2 \geq c\left(\zeta^{-\nu}-(\pi / 2)^{-\nu}\right)-1 / 2 \geq c \zeta^{-\nu}
$$

for $\zeta>0$ small enough. By Lemma 6.2. we have $\vartheta^{2}(z)\left(\vartheta^{\prime}(z)\right)^{2} \geq c(1+z)^{-4 / \nu-2} \geq c z^{-4 / \nu-2}$ for $z \geq 1 / 2$. We thus have

$$
\mathbb{E}\left[\exp \left(-\xi^{*} S_{t} \xi\right)\right] \leq \exp \left(-t r_{0}^{\gamma} q_{0} \int_{1 / 2}^{c \zeta^{-\nu}}\left(1-e^{-c|\xi|^{2} z^{-4 / \nu-2}}\right) d z\right) .
$$

But for $z<|\xi|^{\nu /(2+\nu)}$, we have $|\xi|^{2} z^{-4 / \nu-2} \geq 1$, whence $1-e^{-c|\xi|^{2} z^{-4 / \nu-2}} \geq 1-e^{-c}$. Consequently,

$$
\mathbb{E}\left[\exp \left(-\xi^{*} S_{t} \xi\right)\right] \leq \exp \left(-c t\left(\left(c \zeta^{-\nu}\right) \wedge|\xi|^{\nu /(2+\nu)}-1 / 2\right)\right) .
$$

The conclusion follows. 
We are finally able to conclude this subsection.

Proof of Proposition 4.4. We recall that due to [4, p 92], for all $p \geq 1$, there is a constant $C_{p}$ such that for all nonnegative symmetric $A \in M_{2 \times 2}(\mathbb{R})$,

$$
|\operatorname{det} A|^{-p} \leq C_{p} \int_{\xi \in \mathbb{R}^{2}}|\xi|^{4 p-2} e^{-\xi^{*} A \xi} d \xi
$$

We set $d_{t}=\operatorname{det}\left(u_{\zeta}(t) I+\sigma\left(V_{t}^{\epsilon, \zeta}\right)\right)$. Using Lemma 4.6 we have $\sigma\left(V_{t}^{\epsilon, \zeta}\right)=Y_{t} S_{t} Y_{t}^{*}$, whence $d_{t}=$ $\operatorname{det}^{2}\left(Y_{t}\right) \operatorname{det}\left(u_{\zeta}(t)\left(Y_{t}^{*} Y_{t}\right)^{-1}+S_{t}\right)$. Lemma 4.7 and the Cauchy-Schwarz inequality yield

$$
\begin{aligned}
\mathbb{E}\left[d_{t}^{-p}\right] & \leq \mathbb{E}\left[\operatorname{det}\left(Y_{t}\right)^{-2 p} \operatorname{det}\left(u_{\zeta}(t)\left(Y_{t}^{*} Y_{t}\right)^{-1}+S_{t}\right)^{-p}\right] \\
& \leq e^{C_{p} \Gamma_{\epsilon}^{\gamma}} \mathbb{E}\left[\operatorname{det}\left(u_{\zeta}(t)\left(Y_{t}^{*} Y_{t}\right)^{-1}+S_{t}\right)^{-2 p}\right]^{1 / 2} .
\end{aligned}
$$

Thus due to (4.2) and Lemma 4.10, since $\xi^{*}\left(Y_{t}^{*} Y_{t}\right)^{-1} \xi=\left|\left(Y_{t}^{-1}\right)^{*} \xi\right|^{2} \geq|\xi|^{2}$ by Lemma 4.7.

$$
\begin{aligned}
& \mathbb{E}\left[d_{t}^{-p}\right] \leq C_{p} e^{C_{p} \Gamma_{\epsilon}^{\gamma}}\left(\int_{|\xi| \in \mathbb{R}^{2}}|\xi|^{8 p-2} e^{-u_{\zeta}(t)|\xi|^{2}} \mathbb{E}\left[e^{-\xi^{*} S_{t} \xi}\right] d \xi\right)^{1 / 2} \\
& \leq C_{p} e^{C_{p} \Gamma_{\epsilon}^{\gamma}}\left(\int_{|\xi| \in \mathbb{R}^{2}}|\xi|^{8 p-2} \exp \left(-u_{\zeta}(t)|\xi|^{2}-c t\left[|\xi|^{\nu /(2+\nu)} \wedge \zeta^{-\nu}\right]\right) d \xi\right)^{1 / 2} \\
& \leq C_{p} e^{C_{p} \Gamma_{\epsilon}^{\gamma}}\left(\int_{|\xi| \in \mathbb{R}^{2}}|\xi|^{8 p-2} \exp \left(-c t|\xi|^{\nu /(2+\nu)}\right) d \xi\right)^{1 / 2} .
\end{aligned}
$$

To get the last inequality, observe that if $|\xi|^{\nu /(2+\nu)} \geq \zeta^{-\nu}$, then $|\xi|^{2-\nu /(2+\nu)} \geq \zeta^{-4-\nu}$, so that

$$
u_{\zeta}(t)|\xi|^{2}=t \zeta^{4+\nu}|\xi|^{2}=t \zeta^{4+\nu}|\xi|^{\nu /(2+\nu)}|\xi|^{2-\nu /(2+\nu)} \geq t|\xi|^{\nu /(2+\nu)}
$$

Thus for $0<t_{0}<t<T$, we have

$$
\mathbb{E}\left[d_{t}^{-p}\right] \leq C_{t_{0}, p} e^{C_{p} \Gamma_{\epsilon}^{\gamma}}
$$

as desired.

4.3. Upper-bounds of the derivatives. This subsection is devoted to the following estimates.

Proposition 4.11. For all $l \geq 1$, all $p \geq 1$,

$$
\begin{aligned}
& \mathbb{E}\left(\mathbb{I}_{\left\{\sup _{[0, T]}\left|V_{s}^{\epsilon, \zeta}\right| \leq \Gamma_{\epsilon}\right\}} \sup _{[0, T]}\left|V_{s}^{\epsilon, \zeta}\right|_{l}^{p}\right) \leq C_{l, p} e^{C_{l, p} \Gamma_{\epsilon}^{\gamma}}, \\
& \mathbb{E}\left(\mathbb{I}_{\left\{\sup _{[0, T]}\left|V_{s}^{\epsilon, \zeta}\right| \leq \Gamma_{\epsilon}\right\}} \sup _{[0, T]}\left|L V_{s}^{\epsilon, \zeta}\right|_{l}^{p}\right) \leq C_{l, p} \frac{e^{C_{l, p} \Gamma_{\epsilon}^{\gamma}}}{\epsilon^{(l+1) \zeta^{\nu p}}} .
\end{aligned}
$$

Proof. We will use the estimates from [2, Section 4]. In [2, the coefficients are bounded. But, as long as we are on the set $\left\{\sup _{[0, T]}\left|V_{s}^{\epsilon, \zeta}\right| \leq \Gamma_{\epsilon}\right\}$, we do not need to take a supremum over all $w \in \mathbb{R}^{2}$. For a function $\psi=[0, \infty) \times \mathbb{R}^{2} \times[0,1] \times \mathbb{R}_{*} \mapsto \mathbb{R}\left(\right.$ or $\mapsto \mathbb{R}^{2}$ ) which is infinitely differentiable with respect to $z \in \mathbb{R}_{*}$ and to $w \in \mathbb{R}^{2}$, we set, for $\epsilon \in\left(0, \epsilon_{0}\right), l \geq 1$,

$$
\bar{\psi}_{\epsilon}^{l}(t, \rho, z):=\sup _{\left\{|w| \leq \Gamma_{\epsilon}\right\}} \sum_{0 \leq|\beta|+k \leq l}\left|\partial_{w}^{\beta} \partial_{z}^{k} \psi(t, w, \rho, z)\right| .
$$


Let $c(t, w, \rho, z)=A(\vartheta(z))\left(w-v_{t}(\rho)\right) \mathbf{I}_{\zeta}(z)$, for which $\sup _{w \in \mathbb{R}^{2}}\left|\nabla_{w} c(t, w, \rho, z)\right|=|A(\vartheta(z))| \mathbf{I}_{\zeta}(z)$. Due to [2, Lemma 7], we know that

$$
\begin{aligned}
Y_{l}(t) & :=\mathbb{I}_{\left\{\sup _{[0, t]}\left|V_{s}^{\epsilon, \zeta}\right| \leq \Gamma_{\epsilon}\right\}} \sup _{[0, t]}\left|V_{s}^{\epsilon, \zeta}\right|_{l} \\
& \leq \mathbb{I}_{\left\{\sup _{[0, t]}\left|V_{s}^{\epsilon, \zeta}\right| \leq \Gamma_{\epsilon}\right\}} \sup _{[0, t]}\left|V_{s}^{\epsilon, \zeta}\right|+C_{l}\left(1+\sum_{k=1}^{J_{t}} \bar{c}_{\epsilon}^{l}\left(T_{k}, R_{k}, Z_{k}\right)\right)^{l \times l !} \sup _{[0, t]}\left(\mathcal{E}_{s}\right)^{l \times l !},
\end{aligned}
$$

where

$$
\mathcal{E}_{t}=1+C_{l} \sum_{k=1}^{J_{t}}\left|A\left(\vartheta\left(Z_{k}\right)\right)\right| \mathbf{I}_{\zeta}\left(Z_{k}\right) \mathcal{E}_{T_{k}-}=\prod_{k=1}^{J_{t}}\left(1+C_{l}\left|A\left(\vartheta\left(Z_{k}\right)\right)\right| \mathbf{I}_{\zeta}\left(Z_{k}\right)\right) .
$$

First, we prove exactly as in Lemma 4.7 that for all $p \geq 1,0 \leq t \leq T$,

$$
\mathbb{E}\left[\sup _{[0, t]} \mathcal{E}_{s}^{p}\right] \leq e^{C_{p, l} \Gamma_{\epsilon}^{\gamma}} .
$$

Due to Lemma 6.2, since $|A(\theta)| \leq|\theta|$ and since the derivatives of $\mathbf{I}_{\zeta}$ are bounded uniformly with respect to $\zeta$, we have $\bar{c}_{\epsilon}^{l}(t, \rho, z) \leq C_{l}(1+|z|)^{-1 / \nu}\left(\Gamma_{\epsilon}+\left|v_{t}(\rho)\right|\right) \leq C_{l} \Gamma_{\epsilon}(1+|z|)^{-1 / \nu}\left(1+\left|v_{t}(\rho)\right|\right)$. We thus have, using the Cauchy-Schwarz inequality,

$$
\begin{aligned}
\mathbb{E}\left[Y_{l}(t)^{p}\right] & \leq C_{p} \Gamma_{\epsilon}^{p}+C_{p, l} e^{C_{p, l} \Gamma_{\epsilon}^{\gamma}} \Gamma_{\epsilon}^{p l \times l !} \mathbb{E}\left[1+\left(\sum_{k=1}^{J_{t}}\left(1+\left|Z_{k}\right|\right)^{-1 / \nu}\left(1+\left|v_{T_{k}}\left(R_{k}\right)\right|\right)\right)^{2 p l \times l !}\right]^{1 / 2} \\
& \leq C_{p, l} e^{C_{p, l} \Gamma_{\epsilon}^{\gamma}} \mathbb{E}\left[1+X_{t}^{2 p l \times l !}\right]^{1 / 2}
\end{aligned}
$$

where $X_{t}:=\sum_{k=1}^{J_{t}}\left(1+\left|Z_{k}\right|\right)^{-1 / \nu}\left(1+\left|v_{T_{k}}\left(R_{k}\right)\right|\right)$. We now prove that for any $p \geq 1, \mathbb{E}\left[X_{t}^{p}\right] \leq$ $C_{p} e^{C_{p} \Gamma_{\epsilon}^{\gamma}}$, which will end the proof of the first inequality. Using Remark 3.1 one may find a Poisson measure $M$ on $[0, T] \times[0,1] \times \mathbb{R}_{*} \times[0, \infty)$ with intensity measure $d s d \rho d z d u$ such that

$$
\begin{aligned}
X_{t} & =\int_{0}^{t} \int_{0}^{1} \int_{\mathbb{R}_{*}} \int_{0}^{\infty}(1+|z|)^{-1 / \nu}\left(1+\left|v_{s}(\rho)\right|\right) \mathbb{I}_{\left\{u \leq \phi_{\epsilon}^{\gamma}\left(\left|V_{s-}^{\epsilon, \zeta}-v_{t}(\rho)\right|\right)\right\}} \mathbf{I}_{\zeta}(z) M(d s, d \rho, d z, d u) \\
& \leq \int_{0}^{t} \int_{0}^{1} \int_{\mathbb{R}_{*}} \int_{0}^{\infty}(1+|z|)^{-1 / \nu}\left(1+\left|v_{s}(\rho)\right|\right) \mathbb{I}_{\left\{u \leq \Gamma_{\epsilon}^{\gamma}\right\}} M(d s, d \rho, d z, d u)=: \tilde{X}_{t} .
\end{aligned}
$$

A simple computation shows that

$$
\begin{aligned}
\mathbb{E}\left[\tilde{X}_{t}^{p}\right] & \leq \Gamma_{\epsilon}^{\gamma} \int_{0}^{t} d s \int_{0}^{1} d \rho \int_{\mathbb{R}_{*}} d z \mathbb{E}\left[\left(\tilde{X}_{s}+(1+|z|)^{-1 / \nu}\left(1+\left|v_{s}(\rho)\right|\right)\right)^{p}-\tilde{X}_{s}^{p}\right] \\
& \leq C_{p} \Gamma_{\epsilon}^{\gamma} \int_{0}^{t} d s \int_{0}^{1} d \rho \int_{\mathbb{R}_{*}} d z(1+|z|)^{-1 / \nu}\left(1+\left|v_{s}(\rho)\right|\right) \mathbb{E}\left[1+\tilde{X}_{s}^{p}+\left|v_{s}(\rho)\right|^{p}\right] .
\end{aligned}
$$

Since $\int_{\mathbb{R}_{*}}(1+|z|)^{-1 / \nu} d z<\infty$ and since $\int_{0}^{1}\left|v_{t}(\rho)\right|^{q} d \rho=\int_{\mathbb{R}^{2}}|v|^{q} f_{t}(d v) \leq C_{q}$ for all $q \geq 1$ due to (1.7), we conclude that $\mathbb{E}\left[\tilde{X}_{t}^{p}\right] \leq C_{p} \Gamma_{\epsilon}^{\gamma} \int_{0}^{t} \mathbb{E}\left[\tilde{X}_{s}^{p}\right] d s+C_{p} \Gamma_{\epsilon}^{\gamma}$, whence $\mathbb{E}\left[\tilde{X}_{t}^{p}\right] \leq C_{p} \Gamma_{\epsilon}^{\gamma} e^{C_{p} \Gamma_{\epsilon}^{\gamma}} \leq C_{p} e^{C_{p} \Gamma_{\epsilon}^{\gamma}}$ by the Gronwall Lemma. This ends the proof of the first inequality. 
We now prove the second inequality. We use [2, Lemmas 11 and 12]. We introduce the functions

$$
\begin{aligned}
& g(t, w)=1-\frac{1}{\lambda_{\epsilon, \zeta}} \int_{0}^{1} d \rho \int_{\mathbb{R}_{*}} d z \mathbb{I}_{\{|z|<G(\zeta)+1\}} \phi_{\epsilon}^{\gamma}\left(\left|w-v_{t}(\rho)\right|\right)=1-\frac{1}{2 \Gamma_{\epsilon}^{\gamma}} \int_{0}^{1} d \rho \phi_{\epsilon}^{\gamma}\left(\left|w-v_{t}(\rho)\right|\right), \\
& h(t, w, \rho)=\phi_{\epsilon}^{\gamma}\left(\left|w-v_{t}(\rho)\right|\right) .
\end{aligned}
$$

Then by [2, Lemma 11], for $k=1, \ldots, J_{t}$,

$$
\begin{aligned}
\left|L Z_{k}\right|_{l} \leq C_{l} & \left({\overline{(\log h)_{\epsilon}^{l+1}}}_{\left(T_{k}, R_{k}\right)}\right. \\
& \left.\left.+\left(1+\sup _{[0, t]}\left|V_{s}^{\epsilon, \zeta}\right|_{l+1}\right)^{l+1} \sum_{j=k+1}^{J_{t}}\left[\overline{(\log g)}_{\epsilon}^{l+1}\left(T_{j}\right)+{\overline{(\log h)_{\epsilon}^{l+1}}}^{l+1}\left(T_{j}, R_{j}\right)\right]\right)\right) .
\end{aligned}
$$

Making use of Lemma 6.3.(ii), one easily checks that ${\overline{(\log h)_{\epsilon}}}_{\epsilon}^{l}(t, \rho) \leq C_{l} \epsilon^{-l}$ and that that for any multi-index $q=\left(q_{1}, \ldots, q_{l}\right) \in\{1,2\}^{l},\left|\partial_{q}^{l} g_{\epsilon}(t, w)\right| \leq C_{l} \Gamma_{\epsilon}^{-1} \epsilon^{\gamma-l}$. Hence, using the Faa di Bruno formula (6.1) and the fact that $g_{\epsilon}(t, w) \geq 1 / 2$,

Thus for $k=1, \ldots, J_{t}$,

$$
\overline{(\log g)}_{\epsilon}^{l}(t) \leq C_{l} \epsilon^{\gamma-l}
$$

$$
\left|L Z_{k}\right|_{l} \leq C_{l} \epsilon^{-l-1}\left(1+\sup _{[0, t]}\left|V_{s}^{\epsilon, \zeta}\right|_{l+1}\right)^{l+1}\left(1+J_{t}\right) .
$$

We now infer from [2, Lemma 12] that

$$
\begin{array}{r}
\sup _{[0, t]}\left|L V_{s}^{\epsilon, \zeta}\right|_{l} \leq C_{l}\left(1+\sup _{k=1, \ldots, J_{t}}\left|L Z_{k}\right|_{l}\right)\left(1+\sum_{k=1}^{J_{t}} \bar{c}_{\epsilon}^{l}\left(T_{k}, R_{k}, Z_{k}\right)\right)^{l+1} \\
\times\left(1+\sup _{[0, t]}\left|V_{s}^{\epsilon, \zeta}\right|_{l+1}^{l+2}\right)^{l+1} \sup _{[0, t]}^{l+1} \mathcal{E}_{s}^{l+1}
\end{array}
$$

Using the above estimates, we can upperbound $\sup _{[0, t]}\left|L V_{s}^{\epsilon, \zeta}\right|_{l}$ with

$$
C_{l} \epsilon^{-l-1}\left(1+J_{t}\right)\left(1+\sup _{[0, t]}\left|V_{s}^{\epsilon, \zeta}\right|_{l+1}^{(l+1)(l+3)}\right)\left(1+\Gamma_{\epsilon} \sum_{k=1}^{J_{t}}\left|\vartheta\left(Z_{k}\right)\right|\left(1+\left|v_{T_{k}}\left(R_{k}\right)\right|\right)\right)^{l+1} \sup _{[0, t]} \mathcal{E}_{s}^{l+1}
$$

Thus using the Cauchy-Schwarz inequality and similar arguments as in the proof of the first inequality, we get

$$
\mathbb{E}\left[\sup _{[0, t]}\left|L V_{s}^{\epsilon, \zeta}\right|_{l}^{p}\right] \leq C_{l, p} \epsilon^{-p(l+1)} e^{C_{l, p} \Gamma_{\epsilon}^{\gamma}} \mathbb{E}\left[\left(1+J_{t}\right)^{2 p}\right]^{1 / 2} .
$$

Recall now that $J_{t}$ is a Poisson process with rate $\lambda=\lambda_{\epsilon, \zeta}=4(G(\zeta)+1) \Gamma_{\epsilon}^{\gamma} \leq C \Gamma_{\epsilon}^{\gamma} \zeta^{-\nu}$ by $(\mathbf{A}(\gamma, \nu))$. Hence $\mathbb{E}\left[J_{t}^{p}\right] \leq C_{p}\left(\lambda_{\epsilon, \zeta} T+\left(\lambda_{\epsilon, \zeta} T\right)^{p}\right) \leq C_{p} \Gamma_{\epsilon}^{\gamma p} \zeta^{-\nu p}$. The second inequality follows.

4.4. Proof of the formula. We prove a final lemma to compute the norm of $G_{t}^{\epsilon, \zeta}$.

Lemma 4.12. Recall 4.1]. For all $l \geq 1$, all $t \in[0, T]$,

$$
\left|G_{t}^{\epsilon, \zeta}\right|_{l} \leq C_{l} \mathbb{1}_{\left\{\sup _{[0, t]}\left|V_{s}^{\epsilon, \zeta}\right| \leq \Gamma_{\epsilon}\right\}}\left[1+\mathbb{1}_{\left\{\sup _{[0, t]}\left|V_{s}^{\epsilon, \zeta}\right| \geq \Gamma_{\epsilon}-1\right\}}\left(1+J_{t}\right)^{l}\left(\sup _{[0, t]}\left|V_{s}^{\epsilon, \zeta}\right|_{l}^{l}\right)^{l}\right] .
$$


Proof. Using [2, Lemma 8], we have

$$
\left|G_{t}^{\epsilon, \zeta}\right|_{l} \leq\left|G_{t}^{\epsilon, \zeta}\right|+C_{l}\left(\sup _{\{k=1, \ldots, l\}}\left|\Psi^{(k)}\left(\Sigma_{t}^{\epsilon, \zeta}\right)\right|\right)\left|\Sigma_{t}^{\epsilon, \zeta}\right|_{l}^{l} .
$$

By definition of $\Psi$, we see that $\sup _{\{k=1, \ldots, l\}}\left|\Psi^{(k)}(x)\right| \leq C_{l} \mathbb{I}_{\{1 / 4 \leq x \leq 3 / 4\}}$. Next we observe that by definition, $\Sigma_{t}^{\epsilon, \zeta} \in[1 / 4,3 / 4]$ implies $\sup _{[0, t]}\left|V_{s}^{\epsilon, \zeta}\right| \in\left[\Gamma_{\epsilon}-1, \Gamma_{\epsilon}\right]$. Recalling (4.2), we only have to prove that $\left|\Sigma_{t}^{\epsilon, \zeta}\right|_{l} \leq C_{l}\left(1+J_{t}\right)\left(\sup _{[0, t]}\left|V_{s}^{\epsilon, \zeta}\right|_{l}^{l}\right)$. But of course, $\left|\Sigma_{t}^{\epsilon, \zeta}\right|_{l} \leq\left|\Phi_{\epsilon}\left(\left|V_{0}\right|\right)\right|_{l}+$ $\sum_{1}^{J_{t}}\left|\Phi_{\epsilon}\left(\left|V_{T_{k}}^{\epsilon, \zeta}\right|\right)\right|_{l} \leq\left(1+J_{t}\right) \sup _{[0, t]}\left|\Phi_{\epsilon}\left(\left|V_{s}^{\epsilon, \zeta}\right|\right)\right|_{l}$. It only remains to check that for all $s \in[0, T]$, $\left|\Phi_{\epsilon}\left(\left|V_{s}^{\epsilon, \zeta}\right|\right)\right|_{l} \leq C_{l}\left|V_{s}^{\epsilon, \zeta}\right|_{l}^{l}$. But this is an immediate consequence of the chain rule (see [2, Lemma 8]) and the fact that $v \mapsto \Phi_{\epsilon}(|v|)$ has bounded derivative of all orders, uniformly in $\epsilon$.

Finally, we have all the arms in hand to give the

Proof of Theorem 4.1. We apply (4.3) with

$$
F=V_{t}^{\epsilon, \zeta}+\sqrt{u_{\zeta}(t)}\left(\begin{array}{c}
Z_{-1} \\
Z_{0}
\end{array}\right), \quad G=G_{t}^{\epsilon, \zeta} .
$$

We first notice that for $k \geq 1, D_{k} F=D_{k} V_{t}^{\epsilon, \zeta}$, that $D_{-1} F=\sqrt{u_{\zeta}(t)}\left(\begin{array}{l}1 \\ 0\end{array}\right)$ and $D_{0} F=\sqrt{u_{\zeta}(t)}\left(\begin{array}{l}0 \\ 1\end{array}\right)$. We also have $L F=L V_{t}^{\epsilon, \zeta}+\sqrt{u_{\zeta}(t)}\left(\begin{array}{c}L Z_{-1} \\ L Z_{0}\end{array}\right)$. A simple computation shows that $L Z_{0}=Z_{0}$, so that $D_{k}\left(L Z_{0}\right)=\mathbb{I}_{k=0}$ and thus so that $D_{l} D_{k}\left(L Z_{0}\right)=0$. This yields $\left|L Z_{0}\right|_{l}=1+\left|Z_{0}\right|$. By the same way, $\left|L Z_{-1}\right|_{l}=1+\left|Z_{-1}\right|$. Since $u_{\zeta}(t) \leq 1$,

$$
|F|_{l} \leq C_{l}\left(1+\left|V_{t}^{\epsilon, \zeta}\right|_{l}\right), \quad|L F|_{l} \leq 2+\left|Z_{-1}\right|+\left|Z_{0}\right|+\left|L V_{t}^{\epsilon, \zeta}\right|_{l} \quad \text { and } \quad \sigma(F)=u_{\zeta}(t) I+\sigma\left(V_{t}^{\epsilon, \zeta}\right) .
$$

Using (4.3-4.4), we deduce that for $\beta$ a multi-index with length $q$,

$$
\left|\mathbb{E}\left[\partial_{\beta}^{q} \psi(F) G_{t}^{\epsilon, \zeta}\right]\right| \leq C_{q} \mathbb{E}\left[K_{\beta, q}\right]\|\psi\|_{\infty},
$$

where

$$
\begin{aligned}
& K_{\beta, q}= \frac{\left|G_{t}^{\epsilon, \zeta}\right|_{q}\left(1+\sup _{[0, t]}\left|V_{t}^{\epsilon, \zeta}\right|_{q+1}\right)^{13 q}}{\left(\operatorname{det}\left(u_{\zeta}(t) I+\sigma\left(V_{t}^{\epsilon, \zeta}\right)\right)\right)^{3 q-1}}\left[1+\sum_{j=1}^{q} \sum_{k_{1}+\ldots+k_{j} \leq q-j} \prod_{i=1}^{j}\left(2+\left|Z_{-1}\right|+\left|Z_{0}\right|+\left|L V_{t}^{\epsilon, \zeta}\right|_{k_{i}}\right)\right] \\
& \leq C_{q} \mathbb{I}_{\left\{\sup _{[0, t]}\left|V_{s}^{\epsilon, \zeta}\right| \leq \Gamma_{\epsilon}\right\}} \frac{\left(1+\sup _{[0, t]}\left|V_{t}^{\epsilon, \zeta}\right|_{q+1}\right)^{13 q+q^{2}}}{\left(\operatorname{det}\left(u_{\zeta}(t) I+\sigma\left(V_{t}^{\epsilon, \zeta}\right)\right)\right)^{3 q-1}}\left(1+J_{t}^{q} \mathbb{I}_{\left\{\sup _{[0, t]}\left|V_{s}^{\epsilon, \zeta}\right| \geq \Gamma_{\epsilon}-1\right\}}\right) \\
& \times\left[1+\sum_{j=1}^{q} \sum_{k_{1}+\ldots+k_{j} \leq q-j} \prod_{i=1}^{j}\left(2+\left|Z_{-1}\right|+\left|Z_{0}\right|+\left|L V_{t}^{\epsilon, \zeta}\right|_{k_{i}}\right)\right]
\end{aligned}
$$

due to Lemma 4.12. Using the Cauchy the Cauchy-Schwarz inequality, we obtain

$$
\mathbb{E}\left[K_{\beta, q}\right] \leq C_{q} I_{1} I_{2} I_{3} I_{4}
$$


where

$$
\begin{aligned}
& I_{1}=\mathbb{E}\left[\mathbb{I}_{\left\{\sup _{[0, t]}\left|V_{s}^{\epsilon, \zeta}\right| \leq \Gamma_{\epsilon}\right\}}\left(1+\sup _{[0, t]}\left|V_{t}^{\epsilon, \zeta}\right|_{q+1}\right)^{4\left(13 q+q^{2}\right)}\right]^{1 / 4}, \\
& I_{2}=\mathbb{E}\left[\left(\operatorname{det}\left(u_{\zeta}(t) I+\sigma\left(V_{t}^{\epsilon, \zeta}\right)\right)\right)^{-4(3 q-1)}\right]^{1 / 4}, \\
& I_{3}=\mathbb{E}\left[1+J_{t}^{4 q} \mathbb{I}_{\left\{\sup _{[0, t]}\left|V_{s}^{\epsilon, \zeta}\right| \geq \Gamma_{\epsilon}-1\right\}}\right]^{1 / 4}, \\
& I_{4}=\mathbb{E}\left[1+\sum_{j=1}^{q} \sum_{k_{1}+\ldots+k_{j} \leq q-j} \prod_{i=1}^{j}\left(2+\left|Z_{-1}\right|+\left|Z_{0}\right|+\left|L V_{t}^{\epsilon, \zeta}\right| k_{i}\right)^{4} \mathbb{I}_{\left\{\sup _{[0, t]}\left|V_{s}^{\epsilon, \zeta}\right| \leq \Gamma_{\epsilon}\right\}}\right]^{1 / 4} .
\end{aligned}
$$

Making use of Lemmas 4.4 and 4.11, we immediately get, for $0 \leq t_{0} \leq t \leq T$,

$$
I_{1} \leq C_{q} e^{C_{q} \Gamma_{\epsilon}^{\gamma}} \quad \text { and } \quad I_{2} \leq C_{t_{0}, q} e^{C_{q} \Gamma_{\epsilon}^{\gamma}}
$$

Recall now that $J_{t}$ is a Poisson process with rate $4 \Gamma_{\epsilon}^{\gamma}(G(\zeta)+1) \leq C \Gamma_{\epsilon}^{\gamma} \zeta^{-\nu}$, so that $\mathbb{E}\left[J_{t}^{p}\right] \leq$ $C_{p} \Gamma_{\epsilon}^{\gamma p} \zeta^{-\nu p}$ for all $p \geq 1$. Using Proposition 2.17(iii) with some $1 / \eta_{0}<\kappa<\delta$, and the CauchySchwarz inequality, we obtain

$$
\begin{aligned}
I_{3} & \leq C_{q}+C_{q} \mathbb{E}\left[J_{t}^{8 q}\right]^{1 / 8} \mathbb{P}\left[\sup _{[0, t]}\left|V_{s}^{\epsilon, \zeta}\right| \geq \Gamma_{\epsilon}-1\right]^{1 / 8} \\
& \left.\leq C_{q}+C_{q} \Gamma_{\epsilon}^{\gamma q} \zeta^{-\nu q} e^{-4\left(\Gamma_{\epsilon}-1\right)^{\kappa}}\right) \mathbb{E}\left[\sup _{[0, t]} e^{32\left|V_{s}^{\epsilon, \zeta}\right|^{\kappa}}\right]^{1 / 8} \leq C_{q, \kappa}\left(1+\zeta^{-\nu q} e^{-2 \Gamma_{\epsilon}^{\kappa}}\right) .
\end{aligned}
$$

Finally, using Lemma 4.11, we see that for $j=1, \ldots, q$ and $k_{1}+\ldots+k_{j} \leq q-j$,

$$
\begin{aligned}
& \mathbb{E}\left[\prod_{i=1}^{j}\left(2+\left|Z_{-1}\right|+\left|Z_{0}\right|+\left|L V_{t}^{\epsilon, \zeta}\right|_{k_{i}}\right)^{4} \mathbb{I}_{\left\{\sup _{[0, t]}\left|V_{s}^{\epsilon, \zeta}\right| \leq \Gamma_{\epsilon}\right\}}\right]^{1 / 4} \\
\leq & \prod_{i=1}^{j} \mathbb{E}\left[\left(2+\left|Z_{-1}\right|+\left|Z_{0}\right|+\left|L V_{t}^{\epsilon, \zeta}\right| k_{i}\right)^{4 j} \mathbb{I}_{\left\{\sup _{[0, t]}\left|V_{s}^{\epsilon, \zeta}\right| \leq \Gamma_{\epsilon}\right\}}\right]^{1 /(4 j)} \\
\leq & C_{q} \prod_{i=1}^{j} \mathbb{E}\left[1+\left|L V_{t}^{\epsilon, \zeta}\right|_{k_{i}}^{4 j} \mathbb{I}_{\left\{\sup _{[0, t]}\left|V_{s}^{\epsilon, \zeta}\right| \leq \Gamma_{\epsilon}\right\}}\right]^{1 /(4 j)} \leq C_{q} e^{C_{q} \Gamma_{\epsilon}^{\gamma}}\left[\prod_{i=1}^{j}\left(1+\zeta^{-4 j \nu} \epsilon^{-4 j\left(k_{i}+1\right)}\right)\right]^{1 /(4 j)} \\
\leq & C_{q} e^{C_{q} \Gamma_{\epsilon}^{\gamma}}\left[\prod_{i=1}^{j} \zeta^{-4 j \nu} \epsilon^{-4 j\left(k_{i}+1\right)}\right]^{1 /(4 j)} \leq C_{q} e^{C_{q} \Gamma_{\epsilon}^{\gamma} \zeta^{-j \nu} \epsilon^{-q} \leq C_{q} e^{C_{q} \Gamma_{\epsilon}^{\gamma} \zeta^{-q \nu} \epsilon^{-q}}}
\end{aligned}
$$

whence $I_{4} \leq C_{q} e^{C_{q} \Gamma_{\epsilon}^{\gamma} \zeta^{-q \nu} \epsilon^{-q}}$. All this yields

$$
E\left[K_{\beta, q}\right] \leq C_{t_{0}, q, \kappa} e^{C_{q} \Gamma_{\epsilon}^{\gamma}} \zeta^{-q \nu} \epsilon^{-q}\left(1+\zeta^{-\nu q} e^{-2 \Gamma_{\epsilon}^{\kappa}}\right) \leq C_{t_{0}, q, \kappa} e^{C_{q} \Gamma_{\epsilon}^{\gamma}}\left(\zeta^{-q \nu} \epsilon^{-q}+\zeta^{-2 \nu q} e^{-\Gamma_{\epsilon}^{\kappa}}\right)
$$

For the last inequality, we used that $\Gamma_{\epsilon}=[\log (1 / \epsilon)]^{\eta_{0}}$ and that $\gamma \eta_{0}<1<\kappa \eta_{0}$. Theorem 4.1 is checked. 


\section{Conclusion}

We now wish to end the proof of our main result.

Lemma 5.1. Assume that for some $\alpha \in[0,2)$, some $K>0$, for all $\epsilon \in(0,1)$,

$$
\sup _{[0, T] v_{0} \in \mathbb{R}^{2}} f_{s}\left(\operatorname{Ball}\left(v_{0}, \epsilon\right)\right) \leq K \epsilon^{\alpha} .
$$

Then for $\eta \in(0,1-\nu)$ and $p \geq 1$, for $0<t_{0} \leq t \leq T$, for $\epsilon \in\left(0, \epsilon_{0}\right)$ and $\zeta \in(0,1)$, for $q \geq 1$, for all $\xi \in \mathbb{R}^{2}$ with $|\xi| \geq 1$,

$$
\left|\widehat{f}_{t}(\xi)\right|=\left|\mathbb{E}\left[e^{i\left\langle\xi, V_{t}\right\rangle}\right]\right| \leq C_{q, t_{0}, \eta, p}\left[|\xi|^{-q}\left(\epsilon^{-q-\eta} \zeta^{-\nu q}+\epsilon^{p} \zeta^{-2 \nu q}\right)+|\xi|^{\nu+\eta} \epsilon^{\nu+\gamma+\alpha}+|\xi| \epsilon^{-\eta} \zeta^{1-\nu}\right] .
$$

Proof. We have $\left|\widehat{f}_{t}(\xi)\right|=\left|\mathbb{E}\left[e^{i\left\langle\xi, V_{t}\right\rangle}\right]\right|$ by Proposition (2.1)-(ii). We set $X_{t}^{\zeta}:=\sqrt{u_{\zeta}(t)}\left(Z_{-1}, Z_{0}\right)$ for simplicity and write

$$
\begin{aligned}
\left|\widehat{f}_{t}(\xi)\right| \leq & \left|\mathbb{E}\left[e^{i\left\langle\xi, V_{t}\right\rangle}-e^{i\left\langle\xi, V_{t}^{\epsilon}\right\rangle}\right]\right|+\left|\mathbb{E}\left[e^{i\left\langle\xi, V_{t}^{\epsilon}\right\rangle}-e^{i\left\langle\xi, V_{t}^{\epsilon, \zeta}\right\rangle}\right]\right|+\left|\mathbb{E}\left[e^{i\left\langle\xi, V_{t}^{\epsilon, \zeta}\right\rangle}-e^{i\left\langle\xi, V_{t}^{\epsilon, \zeta}+X_{t}^{\zeta}\right\rangle}\right]\right| \\
& +\left|\mathbb{E}\left[e^{i\left\langle\xi, V_{t}^{\epsilon, \zeta}+X_{t}^{\zeta}\right\rangle}\left(1-G_{t}^{\epsilon, \zeta}\right)\right]\right|+\left|\mathbb{E}\left[e^{i\left\langle\xi, V_{t}^{\epsilon, \zeta}+X_{t}^{\zeta}\right\rangle} G_{t}^{\epsilon, \zeta}\right]\right| \\
= & : A_{1}+\ldots+A_{5} .
\end{aligned}
$$

First, we Theorem 4.1 with $\psi(v)=e^{i\langle\xi, v\rangle}$ and the multi-indexes $\beta_{1}=(1, \ldots, 1)$ and $\beta_{2}=(2, \ldots, 2)$ with length $q$, for which $\partial_{\beta_{1}}^{q} \psi(v)=\left(i \xi_{1}\right)^{q} e^{i\langle\xi, v\rangle}$ and $\partial_{\beta_{2}}^{q} \psi(v)=\left(i \xi_{2}\right)^{q} e^{i\langle\xi, v\rangle}$. For any $\kappa \in\left(1 / \eta_{0}, \delta\right)$,

$$
A_{5} \leq C_{q, t_{0}, \kappa}|\xi|^{-q} e^{C_{q} \Gamma_{\epsilon}^{\gamma}}\left(\zeta^{-\nu q} \epsilon^{-q}+\zeta^{-2 \nu q} e^{-\Gamma_{\epsilon}^{\kappa}}\right) \leq C_{q, t_{0}, \eta, p}|\xi|^{-q}\left(\zeta^{-\nu q} \epsilon^{-q-\eta}+\zeta^{-2 \nu q} \epsilon^{p}\right),
$$

because $\Gamma_{\epsilon}=\log (1 / \epsilon)^{\eta_{0}}$ and $\gamma \eta_{0}<1<\kappa \eta_{0}$. Next, by (4.2) and Proposition 2.1\}(iii),

$$
A_{4} \leq \mathbb{P}\left[\sup _{[0, T]}\left|V_{t}^{\epsilon, \zeta}\right| \geq \Gamma_{\epsilon}-1\right] \leq C_{\kappa} e^{-\left(\Gamma_{\epsilon}-1\right)^{\kappa}} \leq C \epsilon^{\nu+\alpha+\gamma} .
$$

We could have chosen any other positive power of $\epsilon$. We also have, since $\left|e^{i\langle\xi, x\rangle}-e^{i\langle\xi, y\rangle}\right| \leq|\xi||x-y|$,

$$
A_{3} \leq|\xi| \mathbb{E}\left[\left|X_{t}^{\zeta}\right|\right] \leq C|\xi| \sqrt{u_{\zeta}(t)} \leq C|\xi| \zeta^{2+\nu / 2}
$$

Proposition 2.1 (iv) (with $\beta=1$ ) implies

$$
A_{2} \leq|\xi| \mathbb{E}\left[\left|V_{t}^{\epsilon, \zeta}-V_{t}^{\epsilon}\right|\right] \leq C|\xi| e^{C \Gamma_{\epsilon}^{\gamma}} \zeta^{1-\nu} \leq C_{\eta}|\xi| \epsilon^{-\eta} \zeta^{1-\nu} .
$$

Finally, we notice that for $\beta \in(0,1]$,

$$
\left|e^{i\langle\xi, x\rangle}-e^{i\langle\xi, y\rangle}\right| \leq \min (|\xi||x-y|, 2) \leq 2^{1-\beta}|\xi|^{\beta}|x-y|^{\beta} .
$$

Hence using Proposition 2.1 (v) with $\beta=\nu+\eta$ (which is smaller than 1),

$$
A_{1} \leq 2^{1-\beta} \mathbb{E}\left[|\xi|^{\nu+\eta}\left|V_{t}^{\epsilon}-V_{t}\right|^{\nu+\eta}\right] \leq C_{\eta}|\xi|^{\nu+\eta} \epsilon^{\nu+\eta+\gamma+\alpha} e^{C_{\eta} \Gamma_{\epsilon}^{\gamma}},
$$

which we can bound by $C_{\eta}|\xi|^{\nu+\eta} \epsilon^{\nu+\gamma+\alpha}$ as usual. To conclude the proof, it suffices to notice that we obviously have $\epsilon^{\nu+\alpha+\gamma} \leq|\xi|^{\nu+\eta} \epsilon^{\nu+\alpha+\gamma}$ and $|\xi| \zeta^{2+\nu / 2} \leq|\xi| \epsilon^{-\eta} \zeta^{1-\nu}$.

Next, we optimize the previous formula. 
Lemma 5.2. Assume that for some $\alpha \in[0,2)$, some $K>0$, for all $\epsilon \in(0,1)$,

$$
\sup _{[0, T] v_{0} \in \mathbb{R}^{2}} f_{s}\left(\operatorname{Ball}\left(v_{0}, \epsilon\right)\right) \leq K \epsilon^{\alpha} .
$$

Assume that $\nu \in(0,1 / 2)$ and that $\gamma>\nu^{2} /(1-2 \nu)$. Define

$$
p(\alpha)=\frac{(\alpha+\gamma)(1-2 \nu)-\nu^{2}}{(\alpha+\gamma+\nu-1) \nu+1}>0 .
$$

Then for all $r \in(0, p(\alpha))$, all $0<t_{0} \leq t \leq T$ and all $\xi \in \mathbb{R}^{2}$,

$$
\left|\widehat{f}_{t}(\xi)\right| \leq C_{r, t_{0}}|\xi|^{-r} \text {. }
$$

Proof. We can assume that $|\xi| \geq 1$, because $f_{t}$ is a probability measure, so that $\left\|\widehat{f}_{t}\right\|_{\infty}=1$. We use Lemma 5.1 with $\epsilon=|\xi|^{-a}$ and $\zeta=|\xi|^{-b}$, for some $a>0, b>0$ such that $a+\nu b=1-\eta_{1}$, for some small $\eta_{1} \in(0,1)$ to be chosen later. We thus get, for some small $\eta \in(0,1-\nu)$ and some large $p \geq 1, q \geq 1$ to be chosen later, for all $|\xi| \geq 1$,

$$
\begin{aligned}
\left|\widehat{f}_{t}(\xi)\right| & \leq C_{q, t_{0}, \eta, p}\left(|\xi|^{-q+a \eta+(a+\nu b) q}+|\xi|^{-q-a p+2 \nu q b}+|\xi|^{\nu+\eta-a(\nu+\gamma+\alpha)}+|\xi|^{1+a \eta-b(1-\nu)}\right) \\
& =C_{q, t_{0}, \eta, p}\left(|\xi|^{-\eta_{1} q+a \eta}+|\xi|^{-q-a p+2 q\left(1-\eta_{1}-a\right)}+|\xi|^{\nu+\eta-a(\nu+\gamma+\alpha)}+|\xi|^{1+a \eta-\left(1-\eta_{1}-a\right)(1 / \nu-1)}\right) \\
& \leq C_{q, t_{0}, \eta, p}\left(|\xi|^{-\eta_{1} q+1}+|\xi|^{q-a p}+|\xi|^{\nu+\eta-a(\nu+\gamma+\alpha)}+|\xi|^{1+a(\eta+1 / \nu-1)-\left(1-\eta_{1}\right)(1 / \nu-1)}\right) .
\end{aligned}
$$

We used here that $0<a \eta \leq 1$ and $1-\eta_{1}-a \leq 1$. Let now $r \in(0, p(\alpha))$. It remains to show that one may find $q \geq 1, p \geq 1, \eta_{1} \in(0,1), \eta \in(0,1-\nu)$ and $a \in\left(0,1-\eta_{1}\right)$ in such a way that

$$
\begin{aligned}
& \eta_{1} q-1 \geq r, \\
& a p-q \geq r, \\
& a(\nu+\gamma+\alpha)-\nu-\eta \geq r, \\
& \left(1-\eta_{1}\right)(1 / \nu-1)-1-a(\eta+1 / \nu-1) \geq r .
\end{aligned}
$$

It suffices to show that (5.3) and (5.4) hold for some $\eta \in(0,1-\nu)$, some $\eta_{1} \in(0,1)$ and some $a \in\left(0,1-\eta_{1}\right)$ small enough. Indeed, it will then suffice to choose $q$ large enough to get (5.1) and then $p$ large enough to obtain (5.2). Hence it suffices to check that there is $a \in(0,1)$ such that

$$
a(\nu+\gamma+\alpha)-\nu>r \quad \text { and } 1 / \nu-2-a(1 / \nu-1)>r .
$$

But setting $a=\left(1-2 \nu+\nu^{2}\right) /[1+\nu(\nu+\gamma+\alpha-1)]$, we get

$$
a(\nu+\gamma+\alpha)-\nu=1 / \nu-2-a(1 / \nu-1)=p(\alpha)>r .
$$

To conclude the proof, it only remains to check that $a \in(0,1)$. Clearly, $a>0$. To check that $a<1$, it suffices to prove that $1-2 \nu+\nu^{2}<1+\nu(\nu-1)$, which always holds for $\nu>0$.

The last preliminary consists of studying the function $\alpha \mapsto p(\alpha)$.

Lemma 5.3. Assume that $\nu \in(0,1 / 2)$ and that $\gamma>\nu^{2} /(1-2 \nu)$.

(i) The map $\alpha \mapsto p(\alpha)$ is increasing on $[0, \infty)$. The function $\alpha \mapsto p(\alpha) / \alpha$ is decreasing on $(0, \infty)$ and $p\left(a_{\gamma, \nu}\right) / a_{\gamma, \nu}=1$, where $a_{\gamma, \nu}$ was defined by (1.5).

(ii) Furthermore, we have, recalling (1.6)

$$
\begin{aligned}
& q_{\gamma, \nu}>1 \quad \Longleftrightarrow a_{\gamma, \nu}>1 \quad \Longleftrightarrow \quad \nu<1 / 3 \text { and } \gamma>\left(2 \nu+2 \nu^{2}\right) /(1-3 \nu), \\
& q_{\gamma, \nu}>2 \quad \Longleftrightarrow a_{\gamma, \nu}>2 \Longleftrightarrow \nu<1 / 4 \text { and } \gamma>\left(6 \nu+3 \nu^{2}\right) /(1-4 \nu) .
\end{aligned}
$$

Observe that $q_{\gamma, \nu}=p\left(2 \wedge a_{\gamma, \nu}\right)$. 
(iii) For $q \in\left(0, q_{\gamma, \nu}\right)$, one may find $n_{0} \geq 1$ and $0=\alpha_{0}<\alpha_{1}<\ldots<\alpha_{n_{0}}$ such that for all $k \in\left\{0, \ldots, n_{0}-1\right\}, \alpha_{k} \in[0,2)$ and $\alpha_{k+1}<p\left(\alpha_{k}\right)$, with furthermore $\alpha_{n_{0}} \geq q$, all these quantities depending only on $q, \gamma, \nu$.

Proof. We start with point (i). To show that $p$ is increasing, it suffices to note that its derivative is positive if and only if $(1-2 \nu)[(\gamma+\nu-1) \nu+1]>\nu\left[\gamma(1-2 \nu)-\nu^{2}\right]$, i.e. $1-3 \nu+3 \nu^{2}-\nu^{3}>0$, which always holds for $\nu \in(0,1)$. We also have

$$
\frac{p(\alpha)}{\alpha}=\frac{1-2 \nu}{\alpha \nu+[(\gamma+\nu-1) \nu+1]}+\frac{\gamma(1-2 \nu)-\nu^{2}}{\alpha^{2} \nu+\alpha[(\gamma+\nu-1) \nu+1]},
$$

which is obviously decreasing, because under our assumptions, $1-2 \nu>0, \gamma(1-2 \nu)-\nu^{2}>0$ and $(\gamma+\nu-1) \nu+1>0$. Next, $a_{\gamma, \nu}>0$ is designed to solve $\nu a_{\gamma, \nu}^{2}+\nu(\gamma+\nu+1) a_{\gamma, \nu}=\gamma(1-2 \nu)-\nu^{2}$, whence

$$
\frac{p\left(a_{\gamma, \nu}\right)}{a_{\gamma, \nu}}=\frac{a_{\gamma, \nu}(1-2 \nu)+\gamma(1-2 \nu)-\nu^{2}}{\nu a_{\gamma, \nu}^{2}+\nu(\gamma+\nu+1) a_{\gamma, \nu}+(1-2 \nu) a_{\gamma, \nu}}=1 .
$$

We now prove (ii). Due to (i), we clearly have $a_{\gamma, \nu}>1$ if and only if $p(1) / 1>1$, i.e. $[(1+$ $\left.\gamma)(1-2 \nu)-\nu^{2}\right] /[(\gamma+\nu) \nu+1]>1$, which is equivalent to $\nu>1 / 3$ and $\gamma>\left(2 \nu+2 \nu^{2}\right) /(1-3 \nu)$. By the same way, $a_{\gamma, \nu}>2$ if and only if $p(2) / 2>1$, i.e. $\left[(2+\gamma)(1-2 \nu)-\nu^{2}\right] /[(1+\gamma+\nu) \nu+1]>2$, which is equivalent to $\nu>1 / 4$ and $\gamma>\left(6 \nu+3 \nu^{2}\right) /(1-4 \nu)$. Next we note that we always have $q_{\gamma, \nu}=p\left(a_{\gamma, \nu} \wedge 2\right)$. Thus we have $a_{\gamma, \nu}>2$ if and only if $p(2) / 2>1$ if and only if $q_{\gamma, \nu}>2$. Similarly, $a_{\gamma, \nu}>1$ if and only if $p(1) / 1>1$ if and only if $q_{\gamma, \nu}>1$.

Let us now check point (iii). We fix $q \in\left(0, q_{\gamma, \nu}\right)$.

We first assume that $a_{\gamma, \nu} \leq 2$, whence $q_{\gamma, \nu}=a_{\gamma, \nu}$. We fix $q^{\prime} \in\left(q, q_{\gamma, \nu}\right)$, we observe that due to (i), $p\left(q^{\prime}\right) / q^{\prime}>1$ and we consider $\eta>0$ such that $(1-\eta) p\left(q^{\prime}\right) / q^{\prime}=1$. Then by (i), we deduce that the sequence $\alpha_{0}=0, \alpha_{k+1}=(1-\eta) p\left(\alpha_{k}\right)$ takes its values in $\left[0, q^{\prime}\right] \subset[0,2)$ and increases to $q^{\prime}$. Thus for some $n_{0}, \alpha_{n_{0}} \geq q$. Of course, we have $\alpha_{k+1}<p\left(\alpha_{k}\right)$ for all $k \in\left\{0, \ldots, n_{0}-1\right\}$, so that $\left(\alpha_{0}, \ldots, \alpha_{n_{0}}\right)$ solves our problem.

Next we assume that $a_{\gamma, \nu}>2$, whence $q_{\gamma, \nu}=p(2)>2$. We may assume that $q \in(2, p(2))$. We consider $\eta>0$ such that $(1-\eta) p(2) / 2=1$, whence $(1-\eta) p(\alpha) / \alpha>1$ for all $\alpha \in[0,2)$. Then by (i), the sequence $\alpha_{0}=0, \alpha_{k+1}=(1-\eta) p\left(\alpha_{k}\right)$ takes its values in [0,2) and increases to 2 . Consider now $x \in(0,2)$ such that $p(x)=q$ (recall that $q \in(2, p(2))$ is fixed). Then for $n_{0}$ sufficiently large, we have $\alpha_{n_{0}-1}>x$ and thus $\alpha_{n_{0}-1}<q<p\left(\alpha_{n_{0}-1}\right)$. Hence $\left(\alpha_{0}, \ldots, \alpha_{n_{0}-1}, q\right)$ solves our problem.

Finally, we can give the

Proof of Theorem 1.3. Points (ii) and (iii) follow from (i) and Lemma 5.3. We fix $0<t_{0}<T$ and $q \in\left(0, q_{\gamma, \nu}\right)$. The only thing we have to check is that for all $\xi \in \mathbb{R}^{2}$, all $t \in\left[t_{0}, T\right],\left|\widehat{f}_{t}(\xi)\right| \leq$ $C_{t_{0}, q}(1+|\xi|)^{-q}$. Then the Sobolev and the ball estimate will follow (see Lemma 6.1). By Lemma 5.3. we may consider $n_{0} \geq 1$ and $0=\alpha_{0}<\alpha_{1}<\ldots<\alpha_{n_{0}}$ such that for all $k \in\left\{0, \ldots, n_{0}-1\right\}$, $\alpha_{k} \in[0,2)$ and $\alpha_{k+1}<p\left(\alpha_{k}\right)$, with $\alpha_{n_{0}} \geq q$.

Step 1. First, we apply Lemma 5.2 with $\alpha=\alpha_{0}=0$. Since $\alpha_{1}<p\left(\alpha_{0}\right)$, we deduce that

$$
\sup _{t \in\left[t_{0} / n_{0}, T\right]}\left|\widehat{f}_{t}(\xi)\right| \leq C|\xi|^{-\alpha_{1}} .
$$

By Lemma 6.1, we deduce that $\sup _{\left[t_{0} / n_{0}, T\right]} \sup _{v_{0} \in \mathbb{R}^{2}} f_{t}\left(\operatorname{Ball}\left(v_{0}, \epsilon\right)\right) \leq C_{t_{0}, q} \epsilon^{\alpha_{1}}$.

Step 2. Define now $\left(f_{t}^{1}\right)_{t \in\left[0, T-t_{0} / n_{0}\right]}$ by $f_{t}^{1}=f\left(t+t_{0} / n_{0}\right)$. This is also a weak solution of (1.1), which satisfies the same properties as $\left(f_{t}\right)_{t \in[0, T]}$, and the additionnal property that 
$\sup _{\left[0, T-t_{0} / n_{0}\right]} \sup _{v_{0} \in \mathbb{R}^{2}} f_{t}^{1}\left(\operatorname{Ball}\left(v_{0}, \epsilon\right)\right) \leq C_{t_{0}, q} \epsilon^{\alpha_{1}}$. We thus can apply Lemma 5.2 with $\alpha=\alpha_{1}$ and $r=\alpha_{2}<p\left(\alpha_{1}\right)$, to get

$$
\sup _{t \in\left[2 t_{0} / n_{0}, T\right]}\left|\widehat{f}_{t}(\xi)\right|=\sup _{t \in\left[t_{0} / n_{0}, T-t_{0} / n_{0}\right]}\left|\widehat{f_{t}^{1}}(\xi)\right| \leq C|\xi|^{-\alpha_{2}},
$$

whence $\sup _{\left[2 t_{0} / n_{0}, T\right]} \sup _{v_{0} \in \mathbb{R}^{2}} f_{t}\left(\operatorname{Ball}\left(v_{0}, \epsilon\right)\right) \leq C_{t_{0}, q} \epsilon^{\alpha_{2}}$ by Lemma 6.1

Step 3. Iterating this procedure ( $n_{0}$ times), we deduce that

$$
\sup _{t \in\left[t_{0}, T\right]}\left|\widehat{f}_{t}(\xi)\right| \leq C_{t_{0}, r}|\xi|^{-\alpha_{n_{0}}} .
$$

But $f_{t}$ is a probability measure, so that $\left|\widehat{f}_{t}(\xi)\right| \leq 1$. Thus

$$
\sup _{t \in\left[t_{0}, T\right]}\left|\widehat{f}_{t}(\xi)\right| \leq C_{t_{0}, r}(1+|\xi|)^{-\alpha_{n_{0}}}
$$

which ends the proof since $\alpha_{n_{0}} \geq q$.

\section{Appendix}

Fourier transforms. We first prove an easy result on Fourier transforms. Recall that for $f$ a probability measure on $\mathbb{R}^{2}$ and $\xi \in \mathbb{R}^{2}$, we denote by $\widehat{f}(\xi)=\mathcal{F} f(\xi)=\int_{\mathbb{R}^{2}} e^{i\langle\xi, v\rangle} f(d v)$.

Lemma 6.1. Let $f$ be a probability measure on $\mathbb{R}^{2}$ such that $|\widehat{f}(\xi)| \leq K|\xi|^{-\alpha}$, for some $\alpha \in(0,2)$. Then for all $v_{0} \in \mathbb{R}^{2}$, all $\epsilon \in(0,1)$, one has $f\left(\operatorname{Ball}\left(v_{0}, \epsilon\right)\right) \leq C_{K, \alpha} \epsilon^{\alpha}$.

Proof. We use the Plancherel identity. Recall that

$$
\mathcal{F}\left(\mathbb{1}_{\left[x_{0}-\epsilon, x_{0}+\epsilon\right] \times\left[y_{0}-\epsilon, y_{0}+\epsilon\right]}\right)\left(\xi_{1}, \xi_{2}\right)=4 e^{i \xi_{1} x_{0}+i \xi_{2} y_{0}} \sin \left(\xi_{1} \epsilon\right) \sin \left(\xi_{2} \epsilon\right) /\left(\xi_{1} \xi_{2}\right) .
$$

Setting $v_{0}=\left(x_{0}, y_{0}\right)$,

$$
\begin{aligned}
f\left(\operatorname{Ball}\left(v_{0}, \epsilon\right)\right) & \leq \int_{\mathbb{R}^{2}} f(d v) \mathbb{I}_{\left[x_{0}-\epsilon, x_{0}+\epsilon\right] \times\left[y_{0}-\epsilon, y_{0}+\epsilon\right]}(v) \leq C \int_{\mathbb{R}^{2}}\left|\widehat{f}(\xi) \frac{\sin \left(\xi_{1} \epsilon\right) \sin \left(\xi_{2} \epsilon\right)}{\xi_{1} \xi_{2}}\right| d \xi \\
& \leq C_{K} \int_{\mathbb{R}^{2}}|\xi|^{-\alpha} \frac{\left|\sin \left(\xi_{1} \epsilon\right) \sin \left(\xi_{2} \epsilon\right)\right|}{\left|\xi_{1} \xi_{2}\right|} d \xi \leq C_{K} \int_{\mathbb{R}^{2}} \frac{\left|\sin \left(\xi_{1} \epsilon\right) \sin \left(\xi_{2} \epsilon\right)\right|}{\left|\xi_{1} \xi_{2}\right|^{1+\alpha / 2}} d \xi,
\end{aligned}
$$

because $|\xi| \geq \sqrt{2\left|\xi_{1} \xi_{2}\right|}$. We handle the substitution $\xi=x / \epsilon$ and get

$$
f\left(\operatorname{Ball}\left(v_{0}, \epsilon\right)\right) \leq C_{K} \epsilon^{\alpha} \int_{\mathbb{R}^{2}} \frac{\left|\sin \left(x_{1}\right)\right|}{\left|x_{1}\right|^{1+\alpha / 2}} \frac{\left|\sin \left(x_{2}\right)\right|}{\left|x_{2}\right|^{1+\alpha / 2}} d x \leq C_{K} \epsilon^{\alpha}\left(\int_{\mathbb{R}} \frac{\left|\sin \left(x_{1}\right)\right|}{\left|x_{1}\right|^{1+\alpha / 2}} d x_{1}\right)^{2} .
$$

We easily conclude, since $\alpha \in(0,2)$.

Derivatives. We recall here the Faa di Bruno formula. Let $l \geq 1$ be fixed. The exist some coefficients $a_{i_{1}, \ldots, i_{r}}^{l, r}>0$ such that for $\phi: \mathbb{R} \mapsto \mathbb{R}$ and $\tau: \mathbb{R} \mapsto \mathbb{R}$ of class $C^{l}(\mathbb{R})$,

$$
[\phi(\tau)]^{(l)}=\left[\tau^{\prime}\right]^{l} \phi^{(l)}(\tau)+\sum_{r=1}^{l-1}\left(\sum_{i_{1}+\ldots+i_{r}=l} a_{i_{1}, \ldots, i_{r}}^{l, r} \prod_{j=1}^{r} \tau^{\left(i_{j}\right)}\right) \phi^{(r)}(\tau),
$$

where the sum is taken over $i_{1} \geq 1, \ldots, i_{r} \geq 1$ with $i_{1}+\ldots+i_{r}=l$. 
We carry on with another formula. For $l \geq 2$ fixed, there exist some coefficients $c_{i_{1}, ., i_{q}}^{l, r} \in \mathbb{R}$ such that for $\phi: \mathbb{R} \mapsto \mathbb{R}$ a $C^{l}$-diffeomorphism and for $\tau$ its inverse function,

$$
\tau^{(l)}=\sum_{r=l+1}^{2 l-1} \frac{1}{\left(\phi^{\prime}(\tau)\right)^{r}} \sum_{i_{1}+\ldots+i_{q}=r-1} c_{i_{1}, \ldots, i_{q}}^{l, r} \prod_{j=1}^{q} \phi^{\left(i_{j}\right)}(\tau),
$$

where the sum is taken over $q \in \mathbb{N}$, over $i_{1}, \ldots, i_{q} \in\{2, \ldots, l\}$ with $i_{1}+\ldots+i_{q}=r-1$. This formula can be checked by induction on $l \geq 2$.

Regularity of the modified cross section. Recall that $\vartheta:[0, \infty) \mapsto(0, \pi / 2]$ was defined in Section 3 as the inverse of $G:(0, \pi / 2] \mapsto[0, \infty)$ given by $G(x)=\int_{x}^{\pi / 2} b(\theta) d \theta$.

Lemma 6.2. The function $\vartheta$ is $C^{\infty}$ on $(0, \infty)$. For all $z>0$,

$$
\begin{aligned}
\text { (i) } & c(1+z)^{-1 / \nu} \leq \vartheta(z) \leq C(1+z)^{-1 / \nu}, \\
\text { (ii) } & c(1+z)^{-1 / \nu-1} \leq\left|\vartheta^{\prime}(z)\right| \leq C(1+z)^{-1 / \nu-1}, \\
\text { (iii) } & \left|\vartheta^{(k)}(z)\right| \leq C_{k}(1+z)^{-1 / \nu-1}, \quad k \geq 1, \\
\text { (iv) } & \left|(A(\vartheta(z)))^{(k)}\right| \leq C_{k}(1+z)^{-1 / \nu-1}, \quad k \geq 1 .
\end{aligned}
$$

Proof. Due to $(\mathbf{A}(\gamma, \nu))$, we have $c\left(x^{-\nu}-(\pi / 2)^{-\nu}\right) \leq G(x) \leq C\left(x^{-\nu}-(\pi / 2)^{-\nu}\right)$, for all $x \in$ $(0, \pi / 2]$. Since $\vartheta$ is nonincreasing, we easily deduce that for all $z \in[0, \infty),\left(z / c+(\pi / 2)^{-\nu}\right)^{-1 / \nu} \leq$ $\vartheta(z) \leq\left(z / C+(\pi / 2)^{-\nu}\right)^{-1 / \nu}$ and (i) follows. Next, we have $\left|\vartheta^{\prime}(z)\right|=1 /|b(\vartheta(z))|$. But $b(x) \in$ $\left[c x^{-1-\nu}, C x^{-1-\nu}\right]$, so that $\left|\vartheta^{\prime}(z)\right| \in\left[\vartheta^{1+\nu}(z) / C, \vartheta^{1+\nu}(z) / c\right]$. Using (i), we deduce (ii). Next, (iii) is obtained from (6.2): using that for any $k \geq 2,\left|G^{(k)}(x)\right|=\left|b^{(k-1)}(x)\right| \leq C_{k}|x|^{-\nu-k}$, we get

$$
\left|\vartheta^{(k)}(z)\right| \leq C_{k} \sum_{r=k+1}^{2 k-1}|\vartheta(z)|^{r(\nu+1)} \sum_{i_{1}+\ldots+i_{q}=r-1}|\vartheta(z)|^{-\nu q-r+1}
$$

Since we have $i_{1}, \ldots, i_{q} \in\{2, \ldots, k\}$ such that $i_{1}+\ldots+i_{q}=r-1$, we see that $q \leq(r-1) / 2$. Consequently, for $k \geq 2$,

$$
\begin{aligned}
\left|\vartheta^{(k)}(z)\right| & \leq C_{k} \sum_{r=k+1}^{2 k-1}|\vartheta(z)|^{r(\nu+1)}|\vartheta(z)|^{-\nu(r-1) / 2-r+1} \\
& =C_{k} \sum_{r=k+1}^{2 k-1}|\vartheta(z)|^{(r+1) \nu / 2+1} \leq C_{k}|\vartheta(z)|^{(k+2) \nu / 2+1} \leq C_{k}(1+|z|)^{-1 / \nu-1},
\end{aligned}
$$

where we finally used (i). Since $\left|A^{(l)}(\theta)\right| \leq C_{l}$ for all $l \geq 1$, (iv) follows from (6.1) and (iii).

Regularity of the cutoff function. We now prove some regularity properties of our cutoff function $\phi_{\epsilon}$.

Lemma 6.3. Consider the function $\phi_{\epsilon}$ introduced in (2.2).

(i) For $\beta \in(0,1]$, for all $x, y \geq 0$, all $\epsilon \in\left(0, \epsilon_{0}\right)$,

$$
x^{\beta}\left|\phi_{\epsilon}^{\gamma}(x)-\phi_{\epsilon}^{\gamma}(y)\right| \leq C_{\beta} \Gamma_{\epsilon}^{\gamma}|x-y|^{\beta} .
$$

(ii) For every $l \geq 1$, for every multi-index $q=\left(q_{1}, \ldots, q_{l}\right) \in\{1,2\}^{l}$,

$$
\begin{aligned}
& \left|\partial_{v_{q_{l}}} \ldots \partial_{v_{q_{1}}}\left[\log \phi_{\epsilon}(|v|)\right]\right| \leq C_{l}\left(\mathbb{I}_{\left\{|v| \in\left(\epsilon, \Gamma_{\epsilon}-1\right]\right\}}|v|^{-l}+\mathbb{I}_{|v| \in\left(\Gamma_{\epsilon}-1, \Gamma_{\epsilon}+1\right)} \Gamma_{\epsilon}^{-1}\right), \\
& \left|\partial_{v_{q_{l}}} \ldots \partial_{v_{q_{1}}}\left[\phi_{\epsilon}^{\gamma}(|v|)\right]\right| \leq C_{l}\left(\mathbb{I}_{\left\{|v| \in\left(\epsilon, \Gamma_{\epsilon}-1\right]\right\}}|v|^{\gamma-l}+\mathbb{I}_{|v| \in\left(\Gamma_{\epsilon}-1, \Gamma_{\epsilon}+1\right)} \Gamma_{\epsilon}^{\gamma-1}\right) .
\end{aligned}
$$


Proof. We first prove (i). We recall that for any $a, b>0$, there are some constants $0<c_{a, b}<C_{a, b}$ such that for any $x, y \geq 0, c_{a, b}\left|x^{a+b}-y^{a+b}\right| \leq\left(x^{a}+y^{a}\right)\left|x^{b}-y^{b}\right| \leq C_{a, b}\left|x^{a+b}-y^{a+b}\right|$. We also recall that $\phi_{\epsilon}$ is globally Lipschitz continuous with constant 1 , that $\phi_{\epsilon}(x)=\Gamma_{\epsilon}$ for $x \geq \Gamma_{\epsilon}+1$ and that $\phi_{\epsilon}(x) \geq x / 2$ for $x \in\left[0, \Gamma_{\epsilon}+1\right]$, since $\phi_{\epsilon}(x) \geq x$ for $x \in\left[0, \Gamma_{\epsilon}-1\right]$ and since $\phi_{\epsilon}$ is non-decreasing. We set $\Delta_{\epsilon}(x, y)=x^{\beta}\left|\phi_{\epsilon}^{\gamma}(x)-\phi_{\epsilon}^{\gamma}(y)\right|$. If $x, y \geq \Gamma_{\epsilon}+1$, then $\Delta_{\epsilon}(x, y)=0$. If now $x \leq \Gamma_{\epsilon}+1$, then

$$
\begin{aligned}
\Delta_{\epsilon}(x, y) & \leq 2^{\beta} \phi_{\epsilon}^{\beta}(x)\left|\phi_{\epsilon}^{\gamma}(x)-\phi_{\epsilon}^{\gamma}(y)\right| \\
& \leq 2^{\beta}\left(\phi_{\epsilon}^{\beta}(x)+\phi_{\epsilon}^{\beta}(y)\right)\left|\phi_{\epsilon}^{\gamma}(x)-\phi_{\epsilon}^{\gamma}(y)\right| \\
& \leq 2^{\beta} C_{\beta, \gamma}\left|\phi_{\epsilon}^{\beta+\gamma}(x)-\phi_{\epsilon}^{\beta+\gamma}(y)\right| \\
& \leq 2^{\beta} \frac{C_{\beta, \gamma}}{c_{\gamma, \beta}}\left(\phi_{\epsilon}^{\gamma}(x)+\phi_{\epsilon}^{\gamma}(y)\right)\left|\phi_{\epsilon}^{\beta}(x)-\phi_{\epsilon}^{\beta}(y)\right| \\
& \leq 2^{\beta+\gamma} \frac{C_{\beta, \gamma}}{c_{\gamma, \beta}} \Gamma_{\epsilon}^{\gamma}\left|\phi_{\epsilon}(x)-\phi_{\epsilon}(y)\right|^{\beta} \\
& \leq 2^{\beta+\gamma} \frac{C_{\beta, \gamma}}{c_{\gamma, \beta}} \Gamma_{\epsilon}^{\gamma}|x-y|^{\beta} .
\end{aligned}
$$

We used here that $\beta<1$. Finally, if $x \geq \Gamma_{\epsilon}+1$ and $y \leq \Gamma_{\epsilon}+1$,

$$
\begin{aligned}
\Delta_{\epsilon}(x, y) & =x^{\beta}\left|\Gamma_{\epsilon}^{\gamma}-\phi_{\epsilon}^{\gamma}(y)\right| \\
& \leq\left(|x-y|^{\beta}+|y|^{\beta}\right)\left(\Gamma_{\epsilon}^{\gamma}-\phi_{\epsilon}^{\gamma}(y)\right) \\
& \leq|x-y|^{\beta} \Gamma_{\epsilon}^{\gamma}+|y|^{\beta}\left|\phi_{\epsilon}^{\gamma}(x)-\phi_{\epsilon}^{\gamma}(y)\right| \\
& \leq|x-y|^{\beta} \Gamma_{\epsilon}^{\gamma}+2^{\beta+\gamma} \frac{C_{\beta, \gamma}}{c_{\gamma, \beta}} \Gamma_{\epsilon}^{\gamma}|x-y|^{\beta},
\end{aligned}
$$

the last inequality being obtained as previously, since $y \leq \Gamma_{\epsilon}+1$.

To prove (ii), we first observe that for $k \geq 1$,

$$
\left|\phi_{\epsilon}^{(k)}(x)\right| \leq C_{k}\left(\epsilon^{1-k} \mathbb{I}_{\{x \in(\epsilon, 3 \epsilon)\}}+\mathbb{I}_{\{k=1\}} \mathbb{I}_{\left\{x \in\left[3 \epsilon, \Gamma_{\epsilon}-1\right]\right\}}+\mathbb{I}_{\left\{x \in\left(\Gamma_{\epsilon}-1, \Gamma_{\epsilon}+1\right)\right\}}\right) .
$$

Using the Faa di Bruno formula (6.1), one easily deduces that for $l \geq 1$,

$$
\left|\left[\log \phi_{\epsilon}(x)\right]^{(l)}\right| \leq C_{l}\left(\mathbb{1}_{\left\{x \in\left(\epsilon, \Gamma_{\epsilon}\right]\right\}} x^{-l}+\mathbb{1}_{\left\{x \in\left(\Gamma_{\epsilon}-1, \Gamma_{\epsilon}+1\right)\right\}} \Gamma_{\epsilon}^{-1}\right)
$$

and

$$
\left|\left[\phi_{\epsilon}^{\gamma}(x)\right]^{(l)}\right| \leq C_{l}\left(\mathbb{I}_{\left\{x \in\left(\epsilon, \Gamma_{\epsilon}\right]\right\}} x^{\gamma-l}+\mathbb{I}_{\left\{x \in\left(\Gamma_{\epsilon}-1, \Gamma_{\epsilon}+1\right)\right\}} \Gamma_{\epsilon}^{\gamma-1}\right) .
$$

Using again (6.1) and that any derivative of order $k \geq 1$ of $v \mapsto|v|$ is smaller than $C_{k}|v|^{1-k}$, one easily concludes.

Exponential estimates. The next result deals with some estimates concerning the exponential moments for the linearized Boltzmann equation. The study of exponential moments for the nonlinear Boltzmann equation was initiated by Bobylev [5], see also [10] and the references therein. These results really use the nonlinear structure of the Boltzmann equation and we can unfortunately not use them.

Lemma 6.4. For any $\kappa \in(\nu, 1)$, any $v, V \in \mathbb{R}^{2}$, for some constants $C>0, c_{\kappa}>0, C_{\kappa}>0$,

$$
\begin{aligned}
& \int_{-\pi / 2}^{\pi / 2}\left(e^{|V+A(\theta)(V-v)|^{\kappa}}-e^{|V|^{\kappa}}\right) b(\theta) d \theta \leq e^{|V|^{\kappa}}\left[-c_{\kappa} \mathbb{I}_{\{|V| \geq 1,|V| \geq C|v|\}}+C_{\kappa}(|V| \vee 1)^{\kappa+\nu-2} e^{C_{\kappa}|v|^{\kappa}}\right], \\
& \int_{-\pi / 2}^{\pi / 2}\left|e^{|V+A(\theta)(V-v)|^{\kappa}}-e^{|V|^{\kappa}}\right| b(\theta) d \theta \leq C_{\kappa} e^{C_{\kappa}|v|^{\kappa}} e^{C_{\kappa}|V|^{\kappa}} .
\end{aligned}
$$


Proof. We start with the first inequality. Recall that by (1.2), $|A(\theta) V|^{2}=\frac{1+\cos \theta}{2}|V|^{2}$. We also have $\langle V, A(\theta) V\rangle=-\frac{1-\cos \theta}{2}|V|^{2},|A(\theta)| \leq|\theta|$ and $\theta^{2} / 4 \leq 1-\cos \theta \leq \theta^{2}$ for $\theta \in[-\pi / 2, \pi / 2]$. Thus

$$
\begin{aligned}
|V+A(\theta)(V-v)|^{2} & =|V|^{2}+\frac{1-\cos \theta}{2}\left(|V|^{2}+|v|^{2}-2\langle V, v\rangle\right)+2\langle V, A(\theta) V\rangle-2\langle V, A(\theta) v\rangle \\
& =\frac{1+\cos \theta}{2}|V|^{2}+\frac{1-\cos \theta}{2}\left(|v|^{2}-2\langle V, v\rangle\right)-2\langle V, A(\theta) v\rangle \\
& \leq|V|^{2}\left(1-\theta^{2} / 8\right)+\theta^{2}|v|^{2}+4|\theta||V \| v| .
\end{aligned}
$$

An simple computation shows that

$$
|V+A(\theta)(V-v)|^{2} \leq\left\{\begin{array}{lll}
|V|^{2}\left(1-\theta^{2} / 16\right) & \text { if } & |V| \geq 130|v| /|\theta| \\
|V|^{2}+\theta^{2}|v|^{2}+4|\theta||V||v| & \text { if } \quad|V| \leq 130|v| /|\theta|
\end{array}\right\}
$$

In the case where $|V| \leq 1$, we observe that, since $\kappa \in(0,1)$,

$$
|V+A(\theta)(V-v)|^{\kappa} \leq(|V|+|\theta|(|V|+|v|))^{\kappa} \leq|V|^{\kappa}+|\theta|^{\kappa}\left(1+|v|^{\kappa}\right) .
$$

We thus may write

$$
\begin{aligned}
\Delta(V, v):= & \int_{-\pi / 2}^{\pi / 2}\left(e^{|V+A(\theta)(V-v)|^{\kappa}}-e^{|V|^{\kappa}}\right) b(\theta) d \theta \\
\leq & -\int_{-\pi / 2}^{\pi / 2}\left(e^{|V|^{\kappa}}-e^{|V|^{\kappa}\left(1-\theta^{2} / 16\right)^{\kappa / 2}}\right) \mathbb{I}_{\{|\theta| \geq 130|v| /|V|\}} b(\theta) d \theta \\
& +\mathbb{I}_{\{|V| \geq 1\}} \int_{-\pi / 2}^{\pi / 2}\left(e^{\left(|V|^{2}+\theta^{2}|v|^{2}+4|\theta||V||v|\right)^{\kappa / 2}}-e^{|V|^{\kappa}}\right) \mathbb{I}_{\{|\theta| \leq 130|v| /|V|\}} b(\theta) d \theta \\
& +\mathbb{I}_{\{|V| \leq 1\}} \int_{-\pi / 2}^{\pi / 2}\left(e^{|V|^{\kappa}+C_{\kappa}|\theta|\left(1+|v|^{\kappa}\right)}-e^{|V|^{\kappa}}\right) b(\theta) d \theta \\
= & -\Delta_{1}(V, v)+\Delta_{2}(V, v)+\Delta_{3}(V, v) .
\end{aligned}
$$

We now compute carefully. First, we have

$$
\Delta_{1}(V, v) \geq \mathbb{I}_{\{|V| \geq 1,|V| \geq 130|v|\}} \int_{-\pi / 2}^{\pi / 2}\left(e^{|V|^{\kappa}}-e^{|V|^{\kappa}\left(1-\theta^{2} / 16\right)^{\kappa / 2}}\right) \mathbb{I}_{\{|\theta| \geq 1\}} b(\theta) d \theta
$$

But for $|\theta| \geq 1$ and $|V| \geq 1$,

$$
e^{|V|^{\kappa}}-e^{|V|^{\kappa}\left(1-\theta^{2} / 16\right)^{\kappa / 2}} \geq e^{|V|^{\kappa}}-e^{|V|^{\kappa}(1-1 / 16)^{\kappa / 2}} \geq e^{|V|^{\kappa}}\left(1-e^{-|V|^{\kappa}\left(1-(1-1 / 16)^{\kappa / 2}\right)}\right) \geq c_{\kappa} e^{|V|^{\kappa}},
$$

whence, since $b([1, \pi / 2])>0$ by assumption,

$$
\Delta_{1}(V, v) \geq c_{\kappa} \mathbb{I}_{\{|V| \geq 1,|V| \geq 130|v|\}} e^{|V|^{\kappa}} .
$$

Next we observe that for $x, y \geq 0$, since $\kappa / 2 \in(0,1), e^{(x+y)^{\kappa / 2}}-e^{x^{\kappa / 2}} \leq(\kappa / 2) y x^{\kappa / 2-1} e^{x^{\kappa / 2}} e^{y^{\kappa / 2}}$. As a consequence in $\Delta_{2}$, since $|\theta||V| \leq 130|v|$,

$$
\begin{aligned}
e^{\left(|V|^{2}+\theta^{2}|v|^{2}+4|\theta||V||v|\right)^{\kappa / 2}}-e^{|V|^{\kappa}} & \leq C_{\kappa}\left(\theta^{2}|v|^{2}+|\theta||V||v|\right)|V|^{\kappa-2} e^{|V|^{\kappa}} e^{C_{\kappa}\left(\theta^{2}|v|^{2}+|\theta||V||v|\right)^{\kappa / 2}} \\
& \leq C_{\kappa}\left(\theta^{2}|v|^{2}+|\theta||V||v|\right)|V|^{\kappa-2} e^{|V|^{\kappa}} e^{C_{\kappa}|v|^{\kappa}} .
\end{aligned}
$$


Integrating this formula against $b(\theta) d \theta$ (on $|\theta| \in[0, \min (\pi / 2,130|v| /|V|)])$ and using $(\mathbf{A}(\gamma, \nu))$ yields

$$
\begin{aligned}
\Delta_{2}(V, v) \leq & C_{\kappa} \mathbb{I}_{\{|V| \geq 1\}}|V|^{\kappa-2} e^{|V|^{\kappa}} e^{C_{\kappa}|v|^{\kappa}}\left[|v|^{2} \min \left(1,(|v| /|V|)^{2-\nu}\right)+|V||v| \min \left(1,(|v| /|V|)^{1-\nu}\right)\right] \\
\leq & C_{\kappa} \mathbb{I}_{\{|v| \geq|V| \geq 1\}} e^{|V|^{\kappa}} e^{C_{\kappa}|v|^{\kappa}}|V|^{\kappa-2}|v|^{2} \\
& +C_{\kappa} \mathbb{I}_{\{|V| \geq 1,|V| \geq|v|\}} e^{|V|^{\kappa}} e^{C_{\kappa}|v|^{\kappa}}\left(|v|^{4-\nu}|V|^{\kappa+\nu-4}+|v|^{2-\nu}|V|^{\kappa+\nu-2}\right) \\
\leq & C_{\kappa} \mathbb{I}_{\{|V| \geq 1\}}|V|^{\kappa+\nu-2} e^{|V|^{\kappa}} e^{C_{\kappa}|v|^{\kappa}} .
\end{aligned}
$$

We finally used that $\kappa+\nu-4 \leq \kappa-2 \leq \kappa+\nu-2<0$. Recall now that for $x \geq 0, e^{x}-1 \leq x e^{x}$, so that in $\Delta_{3}$, since $|V| \leq 1$,

$$
e^{|V|^{\kappa}+|\theta|^{\kappa}\left(1+|v|^{\kappa}\right)}-e^{|V|^{\kappa}}=e^{|V|^{\kappa}}\left(e^{|\theta|^{\kappa}\left(1+|v|^{\kappa}\right)}-1\right) \leq C_{\kappa}|\theta|^{\kappa} e^{C_{\kappa}|v|^{\kappa}} .
$$

Thus, using $(\mathbf{A}(\gamma, \nu))$ and that $\kappa>\nu$,

$$
\Delta_{3}(V, v) \leq C_{\kappa} \mathbb{I}_{\{|V| \leq 1\}} e^{C_{\kappa}|v|^{\kappa}} \int_{-\pi / 2}^{\pi / 2}|\theta|^{\kappa} b(\theta) d \theta \leq C_{\kappa} \mathbb{I}_{\{|V| \leq 1\}} e^{C_{\kappa}|v|^{\kappa}}
$$

We have proved that

$$
\Delta(V, v) \leq-c_{\kappa} e^{|V|^{\kappa}} \mathbb{1}_{\{|V| \geq 1,|V| \geq 130|v|\}}+C_{\kappa} \mathbb{I}_{\{|V| \geq 1\}}|V|^{\kappa+\nu-2} e^{|V|^{\kappa}} e^{C_{\kappa}|v|^{\kappa}}+C_{\kappa} \mathbb{I}_{\{|V| \leq 1\}} e^{C_{\kappa}|v|^{\kappa}},
$$

which ends the proof of the first inequality.

The second inequality is much easier. Since $\kappa \in(0,1)$, we have for all $x, y \geq 0$,

$$
\left|e^{x^{\kappa}}-e^{y^{\kappa}}\right| \leq \kappa\left|x^{\kappa}-y^{\kappa}\right| e^{(x \vee y)^{\kappa}} \leq|x-y|^{\kappa} e^{(x \vee y)^{\kappa}} .
$$

Thus, since $|A(\theta)| \leq|\theta| \leq \pi / 2$,

$$
\left|e^{|V+A(\theta)(V-v)|^{\kappa}}-e^{|V|^{\kappa}}\right| \leq|\theta|^{\kappa}(|V|+|v|)^{\kappa} e^{(|V|+2|\theta|(|V|+|v|))^{\kappa}} \leq C_{\kappa}|\theta|^{\kappa} e^{C_{\kappa}|V|^{\kappa}} e^{C_{\kappa}|v|^{\kappa}} .
$$

Since $\int_{-\pi / 2}^{\pi / 2}|\theta|^{\kappa} b(\theta) d \theta<\infty$ by $(\mathbf{A}(\gamma, \nu))$, the second inequality holds true.

\section{REFERENCES}

[1] R. Alexandre, L. Desvillettes, C. Villani, B. Wennberg, Entropy dissipation and long-range interactions, Arch. Rat. Mech. Anal. (2000), 152, 327-355.

[2] V. Bally, E. Clément, Integration by parts formula and applications to equations with jumps, Preprint, 2009.

[3] A. Bhatt, R. Karandikar, Invariant measures and evolution equations for Markov processes characterized via martingale problems, Ann. Probab. (1993), 21, No 4, 22462268.

[4] K. Bichteler, J.B. Gravereaux, J. Jacod, Malliavin calculus for processes with jumps, Stochastics Monographs, 2. Gordon and Breach Science Publishers, New York, 1987.

[5] A. V. Bobylev, Moment inequalities for the Boltzmann equation and applications to spatially homogeneous problems, J. Statist. Phys. (1997), 88, no. 5-6, 11831214.

[6] L. Desvillettes, B. Wennberg, Smoothness of the Solution of the Spatially Homogeneous Boltzmann Equation without Cutoff., Comm. Partial Differential Equations (2004), vol. 29, n.1 et 2, 133-155.

[7] N. Fournier, Existence and regularity study for $2 D$ Boltzmann equation without cutoff by a probabilistic approach, Ann. Appl. Probab. (2000), 10, 434-462.

[8] N. Fournier, A new regularization possibility for the Boltzmann equation with soft potentials, Kinet. Relat. Models (2008), 1, 405-414.

[9] N. Fournier, H. GUÉRIn, On the uniqueness for the spatially homogeneous Boltzmann equation with a strong angular singularity, J. Stat. Phys. (2008), 131 (4), 749-781.

[10] N. Fournier, C. Мочнот, On the well-posedness of the spatially homogeneous Boltzmann equation with a moderate angular singularity, Comm. Math. Phys. (2009) 289, 803-824.

[11] C. Graham, S. MÉLÉARD, Existence and regularity of a solution of a Kac equation without cutoff using the stochastic calculus of variations, Comm. Math. Phys. (1999), 205, no. 3, 551-569. 
[12] C. Mounot, C. Villani, Regularity theory for the spatially homogeneous Boltzmann equation with cut-off, Arch. Rational Mech. Anal. (2004), vol. 173, 169-212.

[13] H. Tanaka, Probabilistic treatment of the Boltzmann equation of Maxwellian molecules, Z. Wahrsch. und Verw. Gebiete (1978/79), 46, 67-105.

[14] C. Villani, On a new class of weak solutions to the spatially homogeneous Boltzmann and Landau equations, Arch. Rational Mech. Anal. (1998), 143, 273-307.

[15] C. Villani, Regularity estimates via the entropy dissipation for the spatially homogeneous Boltzmann equation without cut-off, Rev. Matem. Iberoam. (1999), 15, 335-352.

[16] C. Villani, A review of mathematical topics in collisional kinetic theory, Handbook of mathematical fluid dynamics, Vol. I, 71-305, North-Holland, Amsterdam, 2002.

[17] C. Villani, Topics in Optimal Transportation, Graduate Studies in Mathematics Vol. 58, AMS 2003.

V. Bally: Lama UMR 8050, Université Paris Est, Cité Descartes, 5 boulevard Descartes, Champs Sur Marne, 77454 Marne la Vallé Cedex, France, vlad.bally@uniV-mlv.Fr

N. Fournier: LAMA UMR 8050, Université Paris Est, Faculté de Sciences et Technologies, 61, avenue du Général de Gaulle, 94010 Créteil Cedex, France, nicolas.fournier@univ-Paris12.fr 